\title{
An Efficient Dynamic Auction for Heterogeneous Commodities
}

\author{
By Lawrence M. Ausubel*
}

\begin{abstract}
This article proposes a new dynamic design for auctioning multiple heterogeneous commodities. An auctioneer wishes to allocate $K$ types of commodities among $n$ bidders. The auctioneer announces a vector of current prices, bidders report quantities demanded at these prices, and the auctioneer adjusts the prices. Units are credited to bidders at the current prices as their opponents' demands decline, and the process continues until every commodity market clears. Bidders, rather than being assumed to behave as price-takers, are permitted to strategically exercise their market power. Nevertheless, the proposed auction yields Walrasian equilibrium prices and, as from a Vickrey-Clarke-Groves mechanism, an efficient allocation. (JEL D44)
\end{abstract}

In earlier work (Ausubel, 1997, 2004), I proposed an efficient ascending auction design for multiple homogeneous items. In environments where bidders have pure private values and diminishing marginal values, this dynamic auction yields outcomes coinciding with that of the (sealed-bid) Vickrey auction (William Vickrey, 1961), but offers advantages of simplicity, transparency, and privacy preservation. Moreover, in some environments where bidders have interdependent values for the items, this dynamic auction continues to yield efficient outcomes and thus outperforms even the Vickrey auction.

However, situations abound in diverse industries in which heterogeneous (but related) commodities are auctioned. On a typical Monday,

\footnotetext{
* Department of Economics, University of Maryland, Tydings Hall, Room 3105, College Park, MD 20742 (e-mail: ausubel@econ.umd.edu). I am grateful to Kathleen Ausubel, Ken Binmore, Peter Cramton, John Ledyard, Preston McAfee, Paul Milgrom, Thayer Morrill, Ennio Stacchetti, Jeroen Swinkels, Daniel Vincent, three anonymous referees, and participants in the Heidelberg Conference on Auctions and Market Structure, the Stony Brook Multi-Unit Auctions Workshop, the 2000 World Congress of the Econometric Society, the 2001 NBER General Equilibrium Conference, the 2001 NSF Decentralization Conference, and numerous university seminars for helpful comments. Intellectual Property Disclosure: The auction design introduced in this article may be subject to issued or pending patents, in particular, U.S. Patent No. 6,026,383 and U.S. Patent Application No. 09/898,483. Upon request, the author will grant a royalty-free license to the referenced properties for noncommercial research on this auction design.
}

the U.S. Treasury sells in excess of $\$ 10$ billion in three-month bills and $\$ 10$ billion in sixmonth bills. ${ }^{1}$ Current practice is to auction the three-month and six-month bills separately in two independent sealed-bid auctions. In the European UMTS/IMT-2000 spectrum auctions, governments sold both paired and unpaired $3 \mathrm{G}$ spectrum, located at similar frequencies but apparently exhibiting markedly different values. Some governments auctioned these together in fixed bundles, while other governments auctioned the paired spectrum followed by the (less valuable) unpaired spectrum. In the Electricité de France (EDF) generation capacity auctions that have operated quarterly since 2001, as well as separately in the Electrabel virtual power plant auctions that have operated quarterly in Belgium since 2003, the companies sell baseload electricity contracts and peak-load electricity contracts, of at least five different durations each, simultaneously in one dynamic auction procedure (see Ausubel and Peter Cramton, 2004a).

The current article proposes an efficient dynamic auction method for heterogeneous items. The starting point for the new design is a venerable trading procedure, often associated in general equilibrium theory with the fictitious

\footnotetext{
${ }^{1}$ For example, on 25 July 2005, the U.S. Treasury auctioned $\$ 19$ billion in 13-week Treasury bills and $\$ 17$ billion in 26-week bills (Press Release, Department of the Treasury, Bureau of the Public Debt, www.publicdebt.treas. gov).
} 
Walrasian auctioneer, and sometimes implemented in modern times as a dynamic clock auction. An auctioneer wishes to allocate $K$ types of heterogeneous commodities among $n$ bidders. The auctioneer announces a price vector, $\mathbf{p}$, and bidders respond by reporting the quantity vectors that they wish to transact at these prices. The auctioneer then calculates the excess demand and increases or decreases each coordinate of the price vector according to whether the excess demand is positive or negative (Walrasian tâtonnement). This iterative process continues until a price vector is reached at which excess demand is zero, and trades occur only at the final price vector.

In both the fictitious Walrasian auctioneer construct and in most real-world dynamic clock auctions, bidders' payments are linear in the quantities awarded: if bidder $i$ wins quantity vector $\mathbf{q}_{\mathbf{i}}$ in an auction with final price vector $\mathbf{p}$, bidder $i$ pays $\mathbf{p} \cdot \mathbf{q}_{\mathbf{i}}$. Unfortunately, a strategic agent who faces linear prices in the auction then possesses an incentive to underreport her true demand at the announced prices, and this incentive increases in her market share. This is most straightforwardly seen in the case of homogeneous goods (i.e., $K=1$ ), where there is a growing body of both theoretical arguments and empirical evidence. (See detailed discussions for sealed-bid auctions in Ausubel and Cramton, 2002, and for ascending auctions in Ausubel, 2004.) Consequently, when agents have market power, the Walrasian auction procedure typically does not result in Walrasian outcomes. The current article circumvents this problem by extending and generalizing an approach introduced in Ausubel (1997, 2004): units are credited to bidders at the current prices whenever the opposing bidders' demands decline. Specified properly, this nonlinear pricing rule restores the incentive for strategic bidders to bid as price-takers, yielding efficient outcomes even when bidders have market power. ${ }^{2}$

\footnotetext{
${ }^{2}$ The extension of the efficient ascending auction of Ausubel $(1997,2004)$ to $K \geq 2$ commodities poses at least two significant obstacles. First, unlike in the homogeneous goods case, a bidder may now wish to increase her demand for a given commodity along the path toward equilibrium, as prices of substitute commodities increase. Thus, units that once appeared to be "clinched" by another bidder may later be "unclinched," and the auction rules need to reflect
}

One of the objectives of the current article is, thus, to provide both a solution to an outstanding theoretical auction question and a new practical auction design. In a recent article, Sushil Bikhchandani and John W. Mamer (1997, pp. 405-06) ask:

"Do there exist simple market mechanisms (i.e., mechanisms that assign a price to each object) which efficiently allocate multiple indivisible objects when market clearing prices exist? ... Whether there are simple incentive compatible market mechanisms which converge to a competitive equilibrium (whenever one exists) under the more general condition that buyers may want to consume more than one object is an open question."

Meanwhile, Faruk Gul and Ennio Stacchetti (2000, p. 69) conclude the introduction of their recent article by stating:

"More importantly, we show that no dynamic auction can reveal sufficient information to implement the Vickrey mechanism if all Gross Substitutes preferences are allowed. Thus, the unit demand case of Demange et al. [1986] and the multiple homogeneous goods case of Ausubel [1997] are the most general environments for which generalizations of the English auction can be used to implement efficient, strategy-proof allocations." 3

In this article, I will put forth an affirmative answer to Bikhchandani and Mamer's question, while disagreeing with the spirit (but not the letter) of Gul and Stacchetti's conclusion. A simple market mechanism is provided: the auc-

this scenario. Second, $K$ simultaneous auctions are effectively conducted, and it is unclear how the progress of one auction should affect the clinching of units in another. Surprisingly, this article establishes that it suffices to calculate independently the crediting of different commodities; the only formal interaction among the $K$ auctions needs to occur through the simultaneous bidding and the price adjustment rule. With these obstacles resolved, an efficient dynamic auction design for heterogeneous commodities emerges.

${ }^{3}$ They also conclude their article: "Finally, we showed that in general, no efficient, dynamic auction can extract enough information to implement any strategy-proof mechanism" (Gul and Stacchetti, 2000, p. 83). 
tioneer announces price vectors, bidders are asked to respond with their naïve demands, and there is no benefit to bidders from strategizing further. Moreover, the dynamic mechanism economizes on information in the sense that bidders need only report their demands at a one-dimensional set of price vectors, and it maintains privacy in the sense that (starting from an initial price vector of zero) bidders avoid the need to report demands at prices above the market-clearing prices.

While the answer provided here may superficially appear to be in conflict with Gul and Stacchetti's conclusion, there is no formal conflict with their theorems. ${ }^{4}$ Indeed, their elegant analysis is utilized as an important input into the current analysis. Gul and Stacchetti limit their attention, however, to a restrictive class of dynamic auctions and then conclude that "no dynamic auction can reveal sufficient information to implement the Vickrey mechanism" (Gul and Stacchetti, 2000, p. 66). The current article's viewpoint is that a limitation to clock auctions that trace a single ascending price trajectory is an unwarranted restriction on the market designer. Moreover, such a restriction runs counter to the long tradition in economics of Walrasian tâtonnement, which allows prices both to ascend and descend. Taking the more expansive view of dynamic auctions in the current article, an environment of multiple heterogeneous commodities becomes amenable to a generalization of the English auction that can be used to implement efficient, strategy-proof allocations. ${ }^{5,6}$

\footnotetext{
${ }^{4}$ The precise statement of Theorem 6 of Gul and Stacchetti (2000) excludes implementing literally the VickreyClarke-Groves (VCG) outcome by a dynamic clock auction which traces a single ascending price trajectory. By contrast, for the case of substitutes and starting from an initial price vector of zero, Theorems 2 and $2^{\prime}$ of the current article yield the outcome of a modified VCG mechanism using a single ascending trajectory, while Theorems 4 and $4^{\prime}$ of the current article yield literally the VCG outcome using potentially $n$ distinct ascending trajectories generated in parallel. Hence, the conflict herein is only with Gul and Stacchetti's interpretation, and not literally with their theorem.

${ }^{5}$ The modified VCG mechanism is also a Clarke-Groves mechanism, and so it is also strategy-proof. Payoffs differ, however, from the standard VCG payoffs by an additive term that depends on other bidders' reports. If the additive term is positive, the modified VCG mechanism may violate
}

The current article will also seek to offer a modern perspective on the Walrasian auctioneer. Indeed, economists have long been hostile toward this modeling device. Kenneth J. Arrow (1959, p. 43) notes at once the motivation for the fictitious auctioneer and the logical problem that the auctioneer creates: "It is not explained whose decision it is to change prices in accordance with [Walrasian tâtonnement]. Each individual participant in the economy is supposed to take prices as given and determine his choices as to purchases and sales accordingly; there is no one left over whose job it is to make a decision on price." Arrow and Frank H. Hahn (1971, p. 322) elaborate that the auctioneer and perfect competition together produce "the paradoxical problem that a perfect competitor changes prices that he is supposed to take as given."

The present research suggests one way out of the paradox. Instead of an implicit fictitious auctioneer, consider an explicit auction mechanism that uses Walrasian tâtonnement for price adjustment and uses the payment rule proposed in this article. Then, economic agents-even though conscious that they can and do change prices-find it in their interest to take prices as given at every moment and to report their true demands relative to the current prices. As a result, the auction reaches the same efficient

a voluntary participation constraint. If the additive term is negative, the modified VCG mechanism will yield lower expected revenues than the VCG mechanism. Thus, there are advantages to obtaining the standard VCG payoffs, but doing so incurs the cost of using the parallel auction procedure with $n$ distinct ascending trajectories.

${ }^{6}$ In Ausubel and Paul R. Milgrom (2002), we take a different approach to these issues and we again obtain an answer that disagrees with the spirit (but not the letter) of Gul and Stacchetti's conclusion. In our 2002 article, we consider an ascending auction with package bidding. A bid is a pair comprising a package (i.e., a set) of items and a proposed payment for the entire package. Bidders submit bids iteratively, and successive bids by a bidder for a given package are required to ascend. This is not a clock auction, as the bidders - not the auctioneer-name the prices. Thus, Gul and Stacchetti's Theorem 6 does not apply. Nonetheless, our auction procedure should be viewed as a generalization of the English auction. In Theorem 8 of Ausubel and Milgrom (2002), we prove that, for substitutes preferences, sincere bidding is an equilibrium of the ascending package auction and leads to the same outcome as the VCG mechanism. Thus, a restriction to clock auctions is also needed to reach Gul and Stacchetti's conclusion. 
allocation of goods and the same Walrasian equilibrium price vector as if agents were pricetakers and the fictitious auctioneer were present. However, the agents' payments are generally lower than in the Walrasian model.

One attractive feature of the current approach is that it enables very clean results in strategic models of Walrasian equilibrium. In traditional analyses, it has been necessary either to assume that agents are price-takers (i.e., their behavior is optimal only subject to the mistaken belief that their actions do not affect prices) or to set up the model so that economic agents literally have no market power (e.g., they are players in the continuum economy of Robert J. Aumann, 1964). So, for example, the models of Arrow et al. (1959), Alexander S. Kelso and Vincent P. Crawford (1982), Gul and Stacchetti (2000), and Milgrom (2000) are guaranteed to converge to Walrasian equilibria only if agents are posited to bid "straightforwardly" rather than truly optimally, or if the economy has been replicated sufficiently that no individual has any impact on the outcome. By contrast, the main convergence results (Theorems 2, 4, 2', and $4^{\prime}$ ) of the current article show exact convergence to Walrasian equilibrium under the assumptions that bidders have market power and are fully optimizing, without any caveats whatsoever.

Thus, the current article relates to a variety of strands of the literature. First, it connects most directly with several recent papers seeking to extend or explain my analysis in Ausubel (1997, 2004). Motty Perry and Philip J. Reny (2005) adapt my previous ascending-bid design to environments of homogeneous goods with interdependent values. Bikhchandani and Joseph M. Ostroy (2002, forthcoming) and Bikhchandani et al. (2002) formulate the auction problem as a linear programming problem and reinterpret my homogeneous goods design as a primal-dual algorithm. Second, this article relates to the literature on efficient auction design. This includes the classic work of Vickrey (1961), Edward H. Clarke (1971), and Theodore Groves (1973), who provide static dominant-strategy mechanisms for private values settings, as well as recent papers examining the possibility or impossibility of efficient mechanisms with interdependent values, including Eric S. Maskin (1992), Ausubel (1999), Partha Dasgupta and Maskin (2000), Philippe Jehiel and Benny
Moldovanu (2001) and Perry and Reny (2002). Third, this article also relates to the literature exploring Walrasian equilibrium in auction environments with discrete goods. This includes the early work of Kelso and Crawford (1982), as well as recent work by Bikhchandani and Mamer (1997), Gul and Stacchetti (1999, 2000), and Milgrom (2000). Fourth, this article relates to the literature exploring dynamic package bidding - rather than the clock auction-as a procedure for auctioning heterogeneous items, articles such as Jeffrey S. Banks et al. (1989), David C. Parkes and Lyle H. Ungar (2000), and Ausubel and Milgrom (2002).

Finally, the current article connects with the venerable literature on tâtonnement stability and price adjustment processes, which seeks to understand the forces operating in an economy that may drive it toward an equilibrium. The most famous early attempt to treat convergence to equilibrium was made by Leon Walras (1874). Classical results include articles by Arrow et al. (1959), who demonstrate the global stability of Walrasian tâtonnement under the assumption of gross substitutes, and Herbert Scarf (1960), who provides (nonsubstitutes) counterexamples for which Walrasian tâtonnement fails to converge from any starting point other than the equilibria. Hahn (1982) provides a nice survey of the classical literature on tâtonnement stability. Indeed, one way to view the current article is that it introduces a methodology enabling the economist to convert competitive results on tâtonnement stability into gametheoretic results involving strategic agents. It is hoped that the methodology may ultimately enable us to import significant portions of the existing literature on stability of price adjustment processes into a strategic framework.

The article examines two economic environments, each containing bidders with quasilinear utilities and pure private values. In the first environment, the commodities are perfectly divisible. Price is adjusted as a differential equation using the classic specification of Walrasian tâtonnement, and bidders submit bids in continuous time. Surprisingly, it is unnecessary to assume that bidders display substitutes preferences; strictly concave utility functions are sufficient for obtaining the following results in a continuous environment. If a bidder's opponents bid sincerely, then the bidder's payoff is 
path independent and equals a constant translation of social surplus at the final allocation (Lemma 2), implying that the bidder maximizes her payoff by bidding sincerely (Theorem 1). Hence, sincere bidding by every bidder is an equilibrium, yielding a Walrasian equilibrium price vector and an efficient allocation (Theorem 2). With appropriate choice of the initial price, it yields exactly the Vickrey-ClarkeGroves (VCG) payoff to a given bidder (Theorem 3). Finally, a procedure for $n$ parallel auctions is provided which yields exactly the VCG payoff to all $n$ bidders, starting from any initial price vector (Theorem 4).

In the second environment, the commodities are discrete. Then it becomes necessary to assume that agents have substitutes preferences; otherwise, Walrasian prices might fail to exist (see Kelso and Crawford, 1982; Bikhchandani and Mamer, 1997; Gul and Stacchetti, 1999; Milgrom, 2000). Adjusting prices using a simplification and improvement of Gul and Stacchetti's (2000) Walrasian tâtonnement algorithm for discrete commodities, Theorems $1^{\prime}-4$ ' (analogous to Theorems 1-4) obtain. Moreover, starting from an initial price vector of zero, prices ascend only along the adjustment path, so the auction design exhibits the same advantage of privacy preservation as in the homogeneous goods case; and starting from any initial price vector, prices always converge to an equilibrium (i.e., global stability holds) and convergence occurs in finitely many iterations.

The article is organized as follows. Section I illustrates the new dynamic auction. Section II specifies the model. Sections III and IV develop the auction in generality. Sections V and VI prove theorems for the continuous environment. Section VII specifies a tâtonnement process and proves theorems for the discrete environment. Section VIII concludes. Appendix A contains the proofs of the main lemmas and theorems. Appendix B, summarizing the results of a companion paper (Ausubel, 2005), describes in detail the tâtonnement algorithm for discrete goods used in Section VII.

\section{An Illustration of the Efficient Dynamic Auction}

We illustrate the new dynamic auction procedure for heterogeneous commodities using an
TAble 1-Price and Quantity Vectors For ILLUSTRATIVE EXAMPLE WITH $K=2$

\begin{tabular}{lccc}
\hline \hline Price vector & Bidder 1 & Bidder 2 & Bidder 3 \\
\hline $\mathbf{p}(0)=(3,4)$ & $(5,4)$ & $(5,4)$ & $(5,4)$ \\
$\mathbf{p}(1)=(4,5)$ & $(4,4)$ & $(5,4)$ & $(4,3)$ \\
$\mathbf{p}(2)=(5,7)$ & $(4,3)$ & $(4,4)$ & $(4,1)$ \\
$\mathbf{p}(3)=(6,7)$ & $(4,3)$ & $(4,4)$ & $(3,2)$ \\
$\mathbf{p}(4)=(7,8)$ & $(4,2)$ & $(3,4)$ & $(3,2)$ \\
\hline
\end{tabular}

example in which $K=2$. There are two types of commodities, denoted A and B. Real-world examples fitting this description may include the sale of three-month and six-month Treasury bills, the sale of paired and unpaired telecommunications spectrum, or the sale of base-load and peak-load electricity. Suppose that there are $n=3$ bidders and let the supply vector equal $(10,8)$. The auctioneer initially announces a price vector of $\mathbf{p}(0)=(3,4)$, and subsequently adjusts the price vector to $\mathbf{p}(1)=(4,5), \mathbf{p}(2)=$ $(5,7), \mathbf{p}(3)=(6,7)$, and finally $\mathbf{p}(4)=(7,8)$. The bidders' quantities demanded at these price vectors are shown in Table 1.

The crediting of units to bidders occurs as follows. First, consider Bidder 1. When the price vector advances from $\mathbf{p}(0)=(3,4)$ to $\mathbf{p}(1)=(4,5)$, the sum of the quantity vectors demanded by Bidder 1's opponents decreases from $(10,8)$ to $(9,7)$. Thus, one unit of commodity A and one unit of commodity B can be thought of as becoming available to Bidder 1 at the current price of $\mathbf{p}(1)=(4,5)$. The auction algorithm takes this literally, by crediting one unit of commodity A at a price of four, and one unit of commodity B at a price of five to Bidder 1. Next, consider Bidder 2. When the price vector advances from $\mathbf{p}(0)$ to $\mathbf{p}(1)$, the sum of the quantity vectors demanded by Bidder 2's opponents decreases from $(10,8)$ to $(8,7)$. Thus, two units of commodity $\mathrm{A}$ and one unit of commodity B can be thought of as becoming available to Bidder 2 at the current price. The auction algorithm takes this literally, by crediting two units of commodity $\mathrm{A}$ at a price of four, and one unit of commodity B at a price of five, to Bidder 2. Finally, consider Bidder 3. When the price vector advances from $\mathbf{p}(0)$ to $\mathbf{p}(1)$, the sum of the quantity vectors demanded by Bidder 3's opponents decreases from $(10,8)$ to $(9$, 
Table 2-Credits and Debits for Illustrative Example with $K=2$

\begin{tabular}{|c|c|c|c|}
\hline Price vector & Bidder 1 & Bidder 2 & Bidder 3 \\
\hline $\mathbf{p}(0)=(3,4)$ & Initialization & Initialization & Initialization \\
\hline $\mathbf{p}(1)=(4,5)$ & $\begin{array}{l}1 \text { unit of A credited at } 4 \\
1 \text { unit of B credited at } 5 \\
\text { Cumulative payment }=9\end{array}$ & $\begin{array}{l}2 \text { units of A credited at } 4 \\
1 \text { unit of B credited at } 5 \\
\text { Cumulative payment }=13\end{array}$ & $\begin{array}{l}1 \text { unit of A credited at } 4 \\
0 \text { units of B credited at } 5 \\
\text { Cumulative payment }=4\end{array}$ \\
\hline $\mathbf{p}(2)=(5,7)$ & $\begin{array}{l}1 \text { unit of A credited at } 5 \\
2 \text { units of B credited at } 7 \\
\text { Cumulative payment }=28\end{array}$ & $\begin{array}{l}0 \text { units of A credited at } 5 \\
3 \text { units of B credited at } 7 \\
\text { Cumulative payment }=34\end{array}$ & $\begin{array}{l}1 \text { unit of A credited at } 5 \\
1 \text { unit of B credited at } 7 \\
\text { Cumulative payment }=16\end{array}$ \\
\hline $\mathbf{p}(3)=(6,7)$ & $\begin{array}{l}1 \text { unit of A credited at } 6 \\
1 \text { unit of B debited at } 7 \\
\text { Cumulative payment }=27\end{array}$ & $\begin{array}{l}1 \text { unit of A credited at } 6 \\
1 \text { unit of B debited at } 7 \\
\text { Cumulative payment }=33\end{array}$ & $\begin{array}{l}0 \text { units of A credited at } 6 \\
0 \text { units of B credited at } 7 \\
\text { Cumulative payment }=16\end{array}$ \\
\hline $\mathbf{p}(4)=(7,8)$ & $\begin{array}{l}1 \text { unit of A credited at } 7 \\
0 \text { units of B credited at } 8 \\
\text { Cumulative payment }=34\end{array}$ & $\begin{array}{l}0 \text { units of A credited at } 7 \\
1 \text { unit of B credited at } 8 \\
\text { Cumulative payment }=41\end{array}$ & $\begin{array}{l}1 \text { unit of A credited at } 7 \\
1 \text { unit of B credited at } 8 \\
\text { Cumulative payment }=31\end{array}$ \\
\hline
\end{tabular}

8). Thus, one unit of commodity A and zero units of commodity B can be thought of as becoming available to Bidder 3 at the current price. Again, the auction algorithm takes this literally, by crediting one unit of commodity A at a price of four and zero units of commodity B at a price of five, to Bidder 3 .

The process continues as the price vector advances. One interesting moment occurs when the price advances from $\mathbf{p}(2)=(5,7)$ to $\mathbf{p}(3)=$ $(6,7)$. Observe that Bidder 3's demand vector changes from $(4,1)$ to $(3,2)$, while the other bidders' demand vectors remain constant. In particular, Bidder 3's demand for commodity B increases, meaning that one fewer unit of commodity B remains available for Bidders 1 and 2 . Consequently, the auction algorithm needs to take this literally, by debiting one unit of commodity $\mathrm{B}$ at the current price of seven from each of Bidders 1 and 2.

The entire progression of units credited and debited is summarized in Table 2.

At $\mathbf{p}(4)=(7,8)$, supply and demand are now in balance for both commodities. Thus, $\mathbf{p}(4)$ becomes the final price. Bidders 1,2, and 3 receive their quantity vectors of $(4,2),(3,4)$ and $(3,2)$, respectively, demanded at the final price. Observe that, for each bidder, the quantity vector demanded at the final price equals the sum of all units credited or debited along the way. Since many of the credits and debits occurred at earlier prices, however, bidders' payments do not generally equal their final demands evaluated at the final prices. Rather, the bidders' payments are related to those from the VCG mechanism, justifying the sincere bidding assumed in this section by making it incentive compatible.

\section{The Model}

A seller wishes to allocate units of each of $K$ heterogeneous commodities among a set of $n$ bidders, $N \equiv\{1, \ldots, n\}$. The seller's available supply of commodities is denoted by $\mathbf{S}=\left(S^{1}\right.$, $\left.\ldots, S^{K}\right) \in \mathbb{R}_{++}^{K}$. Bidder $i$ 's consumption set, $X_{i}$, is assumed to be a compact, convex ${ }^{7}$ subset of $\mathbb{R}_{+}^{K}$, and bidder $i$ 's consumption bundle is denoted by $\mathbf{x}_{\mathbf{i}}=\left(x_{i}^{1}, \ldots, x_{i}^{K}\right) \in X_{i}$. The following assumptions are made for the divisible commodities model: ${ }^{8}$

(A1) Pure private values: Bidder i's value, $U_{i}\left(\mathbf{x}_{\mathbf{i}}\right)$, for consumption vector $\mathbf{x}_{\mathbf{i}}$ does not change when bidder $i$ learns other bidders' information.

(A2) Quasilinearity: Bidder i's utility from receiving the consumption vector $\mathbf{x}_{\mathbf{i}}$ in re-

\footnotetext{
${ }^{7}$ More precisely, convexity will be assumed when considering the divisible commodities model emphasized in Sections V and VI; obviously, convexity in $\mathbb{R}^{K}$ will not be assumed for the discrete commodities model studied in Section VII, since then $X_{i} \subset \mathbb{Z}_{+}^{K}$.

${ }^{8}$ In addition, to avoid arcane difficulties, it is assumed that the consumption sets of the bidders and the available supply $\mathbf{S}$ of commodities are such that there exists a feasible allocation of $\mathbf{S}$ among all the bidders and, for each $i$, there exists a feasible allocation of $S$ among the bidders $j \neq i$.
} 
turn for the payment $y_{i}$ is given by $U_{i}\left(\mathbf{x}_{\mathbf{i}}\right)-y_{i}$.

(A3) Monotonicity: The function $U_{i}: X_{i} \rightarrow \mathbb{R}$ is increasing, i.e., if $\mathbf{x}_{\mathbf{i}}^{\prime} \geq \mathbf{x}_{\mathbf{i}}$ and $\mathbf{x}_{\mathbf{i}}^{\prime} \neq \mathbf{x}_{\mathbf{i}}$, then $U\left(\mathbf{x}_{\mathbf{i}}^{\prime}\right)>U\left(\mathbf{x}_{\mathbf{i}}\right)$.

(A4) Concavity: The function $U_{i}: X_{i} \rightarrow \mathbb{R}$ is concave.

Note that, for the divisible commodities model, the concavity assumption (A4) immediately implies continuity: the function $U_{i}: X_{i} \rightarrow \mathbb{R}$ is continuous.

The price vector will be denoted by $\mathbf{p}=$ $\left(p^{1}, \ldots, p^{K}\right) \in \mathbb{R}^{K}$. Bidder $i$ 's indirect utility function, $V_{i}(\mathbf{p})$, and true demand correspondence, $Q_{i}(\mathbf{p})$, are defined respectively by:

$$
\begin{aligned}
& V_{i}(\mathbf{p})=\underset{\mathbf{x}_{\mathbf{i}} \in X_{i}}{\max _{i}}\left\{U_{i}\left(\mathbf{x}_{\mathbf{i}}\right)-\mathbf{p} \cdot \mathbf{x}_{\mathbf{i}}\right\} \text {, and } \\
& Q_{i}(\mathbf{p})=\underset{\mathbf{x}_{\mathbf{i}} \in X_{i}}{\arg \max }\left\{U_{i}\left(\mathbf{x}_{\mathbf{i}}\right)-\mathbf{p} \cdot \mathbf{x}_{\mathbf{i}}\right\} .
\end{aligned}
$$

Observe that $V_{i}(\mathbf{p})$ is well defined and $Q_{i}(\mathbf{p})$ is nonempty. If the correspondence $Q_{i}(\mathbf{p})$ is single valued (as will be the case when we strengthen (A4) to assume strictly concave utility in Sections V and VI, below), then we may also refer to the solution of equation (2) as the demand function $\mathbf{q}_{\mathbf{i}}(\mathbf{p})$.

Since $U_{i}(\cdot)$ is continuous and concave, its conjugate function, $-V_{i}(\cdot): \mathbb{R}^{K} \rightarrow \mathbb{R}$, is continuous, closed, and concave (R. Tyrrell Rockafellar, 1970, Thm. 12.2 and p. 308). We have:

$$
\partial V_{i}(\mathbf{p})=-Q_{i}(\mathbf{p}), \quad \text { for all } \mathbf{p} \in \mathbb{R}^{K},
$$

i.e., $\mathbf{x}$ is a subgradient of $V_{i}$ at $\mathbf{p}$ if and only if $-\mathbf{x}$ is an element of bidder $i$ 's true demand correspondence at $\mathbf{p}$ (Rockafellar, 1970, Thm. 23.5). Note that equation (3) is merely a general version of $\nabla V_{i}(\mathbf{p})=-\mathbf{q}_{\mathbf{i}}(\mathbf{p})$, Roy's identity as restricted to quasilinear utility. To see this, consider the case where $U_{i}(\cdot)$ is twice continuously differentiable and strictly concave. Then demand is a continuously differentiable function, $\mathbf{q}_{\mathbf{i}}(\cdot)$. Furthermore, since $V_{i}(\mathbf{p})=U_{i}\left(\mathbf{q}_{\mathbf{i}}(\mathbf{p})\right)-\mathbf{p} \cdot$ $\mathbf{q}_{\mathbf{i}}(\mathbf{p})$, the Envelope Theorem implies $\nabla V_{i}(\mathbf{p})=$ $-\mathbf{q}_{\mathbf{i}}(\mathbf{p})$.

With every time $t \in[0, \infty)$, we associate a price vector $\mathbf{p}(t)$, and each bidder $i$ selects a bid $\mathbf{x}_{\mathbf{i}}(t)$. We say that bidder $i$ bids sincerely if her bid always belongs to her true demand correspondence:

Sincere bidding. ${ }^{9}$ Bidder $i$ is said to bid sincerely relative to utility function $U_{i}(\cdot)$ if, at every time $t \in[0, \infty)$, her bid $\mathbf{x}_{\mathbf{i}}(t) \in$ $Q_{i}(\mathbf{p}(t))=\arg \max _{\mathrm{x}_{\mathrm{i}} \in X_{i}}\left\{U_{i}\left(\mathbf{x}_{\mathbf{i}}\right)-\mathbf{p}(t) \cdot \mathbf{x}_{\mathbf{i}}\right\}$.

Next, we define two notions of efficient outcomes for this auction environment, the first taken from general equilibrium theory and the second taken from game theory:

DEFINITION 1: A Walrasian equilibrium is a price vector $\mathbf{p}^{*}$ and a profile of consumption bundles $\left\{\mathbf{x}_{\mathbf{i}}^{*}\right\}_{i=1}^{n}$ for bidders such that $\mathbf{x}_{\mathbf{i}}^{*} \in$ $Q_{i}\left(\mathbf{p}^{*}\right)$, for $i=1, \ldots, n$, and $\sum_{i=1}^{n} \mathbf{x}_{\mathbf{i}}^{*}=\mathbf{S}$.

DEFINITION 2: The Vickrey-Clarke-Groves (VCG) mechanism is the following procedure: each bidder $i$ reports a valuation function, $U_{i}$ : $X_{i} \rightarrow \mathbb{R}$, to the auctioneer. The auctioneer assigns a consumption bundle, $\mathbf{x}_{\mathbf{i}}^{*}$, to each bidder $i$ and charges a payment of $y_{i}^{*}=U_{i}\left(\mathbf{x}_{\mathbf{i}}^{*}\right)-$ $W^{*}+W_{-i}^{*}$, where:

$\left\{\mathbf{x}_{\mathbf{i}}^{*}\right\}_{i=1}^{n}$

$$
\begin{aligned}
& \in \arg \max \left\{\sum_{i=1}^{n} U_{i}\left(\mathbf{x}_{\mathbf{i}}\right): \mathbf{x}_{\mathbf{i}} \in X_{i} \text { and } \sum_{i=1}^{n} \mathbf{x}_{\mathbf{i}}=\mathbf{S}\right\}, \\
& W^{*}=\max \left\{\sum_{i=1}^{n} U_{i}\left(\mathbf{x}_{\mathbf{i}}\right): \mathbf{x}_{\mathbf{i}} \in X_{i} \text { and } \sum_{i=1}^{n} \mathbf{x}_{\mathbf{i}}=\mathbf{S}\right\},
\end{aligned}
$$

and

$$
W_{-i}^{*}=\max \left\{\sum_{j \neq i} U_{j}\left(\mathbf{x}_{\mathbf{j}}\right): \mathbf{x}_{\mathbf{j}} \in X_{j} \text { and } \sum_{j \neq i} \mathbf{x}_{\mathbf{j}}=\mathbf{S}\right\} \text {. }
$$

The VCG payoffs (if reports are truthful) are the payoffs $W^{*}-W_{-i}^{*}$.

\footnotetext{
${ }^{9}$ If bidder $i$ 's utility function is strictly concave (as will be assumed in Sections V and VI), then the demand correspondence $Q_{i}(\mathbf{p})$ is single valued, and so sincere bidding simply means that $\mathbf{x}_{\mathbf{i}}(t)=\mathbf{q}_{\mathbf{i}}(\mathbf{p}(t))$ for all $t \in[0, T]$.
} 
Assumptions (A1)-(A4) guarantee the existence of Walrasian equilibrium. Given Assumptions (A1)-(A4), if bidders report truthfully, then the VCG mechanism is also well defined. It is well known that truthful reporting is a dominant strategy equilibrium of the VCG mechanism. By the First Theorem of Welfare Economics, any Walrasian equilibrium allocation $\left\{\mathbf{x}_{i}^{*}\right\}_{i=1}^{n}$ is welfare maximizing, so (apart from nonuniqueness issues $^{10}$ ) the Walrasian and VCG allocations coincide. The payments in the VCG mechanism, however, are generally less than the linear calculation $\mathbf{p}^{*} \cdot \mathbf{x}_{\mathbf{i}}^{*}$ of the Walrasian equilibrium.

Finally, we define a modification of the VCG mechanism that will be useful in characterizing the outcomes of the auction proposed in this article. It is somewhat related to the notion of a Vickrey auction with a reserve price discussed in Ausubel and Cramton (2004b):

DEFINITION 3: The modified VCG mechanism with price of $\boldsymbol{p}(0)$ is the following procedure: each bidder $i$ reports a valuation function, $U_{i}: X_{i} \rightarrow \mathbb{R}$, to the auctioneer. The auctioneer assigns a consumption bundle, $\mathbf{x}_{\mathbf{i}}^{*}$, to each bidder $i$ and charges a payment of $y_{i}^{*}=U_{i}\left(\mathbf{x}_{\mathbf{i}}^{*}\right)-$ $W^{* *}+W_{-i}^{*}$, where:

$$
\begin{array}{r}
\left\{\mathbf{x}_{\mathbf{i}}^{*}\right\}_{i=1}^{n} \in \arg \max \left\{\sum_{i=1}^{n}\left(U_{i}\left(\mathbf{x}_{\mathbf{i}}\right)-\mathbf{p}(0) \cdot \mathbf{x}_{\mathbf{i}}\right):\right. \\
\left.\quad \mathbf{x}_{\mathbf{i}} \in X_{i} \text { and } \sum_{i=1}^{n} \mathbf{x}_{\mathbf{i}}=\mathbf{S}\right\},
\end{array}
$$$$
W^{* *}=\max \left\{\sum_{i=1}^{n}\left(U_{i}\left(\mathbf{x}_{\mathbf{i}}\right)-\mathbf{p}(0) \cdot \mathbf{x}_{\mathbf{i}}\right):\right.
$$

$$
\left.\mathbf{x}_{\mathbf{i}} \in X_{i} \text { and } \sum_{i=1}^{n} \mathbf{x}_{\mathbf{i}}=\mathbf{S}\right\} \text {, and }
$$$$
W_{-i}^{*}=\max \left\{\sum_{j \neq i}\left(U_{j}\left(\mathbf{x}_{\mathbf{j}}\right)-\mathbf{p}(0) \cdot \mathbf{x}_{\mathbf{j}}\right): \mathbf{x}_{\mathbf{j}} \in X_{j}\right\} .
$$

$$
\begin{gathered}
\hat{C}_{i}(t)=\max \left\{0, S-x_{-i}(t)\right\} \quad \text { and } \\
C_{i}(t)=\sup _{\hat{t} \in[0, t]} \hat{C}_{i}(\hat{t}),
\end{gathered}
$$

and we define the payment, $y_{i}(T)$, of bidder $i$ by the following Stieltjes integral:

$$
y_{i}(T)=\int_{0}^{T} p(t) d C_{i}(t) .
$$

This paragraph provides a short review of Stieltjes integrals, drawing from the presentation of Tom M. Apostol (1957). The Stieltjes

\footnotetext{
${ }^{10}$ If we strengthen Assumption (A4) to assume strictly concave utilities (as in Sections V and VI), then the Walrasian and VCG allocations of goods are each unique.
}

The modified VCG mechanism with price of $W^{*}$ in Definition 1: social surplus is calculated with all bidders present and the supply constraint of $\sum_{i=1}^{n} \mathbf{x}_{\mathbf{i}}=\mathbf{S}$ is maintained, but a social costies. The of $\mathbf{p}(0)$. Similar to the regular VCG mechanism, the modified mechanism awards bidder $i$ exthe difference between these two surplus 3 below, if $\mathbf{p}(0)$ happens to be chosen such that the market absent bidder $i$ clears (i.e., $\sum_{j \neq i}$ $\left.\mathbf{q}_{\mathbf{j}}(\mathbf{p}(0))=\mathbf{S}\right)$, then bidder $i$ 's modified VCG payoff coincides with her regular VCG payoff.

\section{III. "Clinching" versus "Crediting and Debiting"}

In Ausubel (1997, 2004), I introduced the notion of "clinching" for auctions of homoge列 quantity and price vectors temporarily reduce to scalars. Let the auction start at time 0 and clear at time $T$, and let $p(t)$ denote the price at time $t \in[0, T]$. Let $x_{i}(t)$ denote bidder $i$ 's demand at demands by all of bidder $i$ 's opponents and let $x_{-i}(t)=\sum_{j \neq i} x_{j}(t)$ denote the aggregate demand of bidder $i$ 's opponents at time $t$. We define the cumulative clinches, $C_{i}(t)$, by: 
integral $\int_{a}^{b} f(t) d \alpha(t)$ involves two functions, $f$ and $\alpha$. When $\alpha$ has a continuous derivative, the Stieltjes integral reduces to the Riemann integral $\int_{a}^{b} f(t) \alpha^{\prime}(t) d t$. The Stieltjes integral may still be evaluated, however, when $\alpha$ is not differentiable or even when $\alpha$ is discontinuous. In particular, if $f$ is continuous on $[a, b]$ and $\alpha$ is of bounded variation on $[a, b]$, then the integral $\int_{a}^{b} f(t) d \alpha(t)$ exists (Apostol, 1957, Theorem 9-26). In turn, $\alpha$ is of bounded variation on [a, $b$ ] if and only if $\alpha$ can be expressed as the difference of two increasing functions. A definition of the Stieltjes integral may be found in Apostol (1957, Definition 9-1).

An example of evaluating a Stieltjes integral of a discontinuous function can be based on the illustrative example of Section I of Ausubel (2004). Five objects are available for auction, and the price is given by $p(t)=t$. Bidder 1's demand, $x_{1}(t)$, equals three at all $t \in[0,103)$. The aggregate demand, $x_{-1}(t)$, of bidder 1's opponents equals: five, at $t \in[49,65)$; four, at $t \in[65,75)$; three, at $t \in[75,85)$; and two, at $t \in[85,125)$. Consequently, the auction clears at $T=85$ and, applying equation (4), we have:

$$
d C_{1}(t)= \begin{cases}1, & \text { if } t=65,75, \text { or } 85 \\ 0, & \text { otherwise }\end{cases}
$$

Thus, the Stieltjes integral, $\int_{0}^{85} p(t) d C_{1}(t)$, evaluates to $p(65) d C_{1}(65)+p(75) d C_{1}(75)+$ $p(85) d C_{1}(85)$, which equals $65+75+85=$ 225.

This example has the following simple interpretation. The function $C_{i}(t)$ indicates the "cumulative clinches," e.g., at the time $t=75$, it has become a foregone conclusion that Bidder 1 will win at least $C_{1}(75)=2$ units of the homogeneous good, since there are five units available and her opponents demand only three. The function $d C_{i}(t)$ indicates the "current clinches," i.e., the number of additional units that the bidder has newly clinched at time $t$. The interpretation of the Stieltjes integral of equation (5) is then that, at every time $t$ when it becomes inevitable that a bidder wins additional units $d C_{i}(t)$, she wins them at the current price $p(t)$. For example, Bidder 1 clinched one unit at 65 , one unit at 75 , and one unit at 85 , for a total payment of 225. In Ausubel (2004, Theorem 1), I proved for homogeneous goods that, if the auction rules provide that each bidder $i$ is assigned $x_{i}(T)$ units and is assessed a payment $y_{i}(T)$ determined by equations (4) and (5), then sincere bidding by all bidders is an (efficient) equilibrium.

It is possible to modify equation (5) in a relatively innocuous way. Note that the expression $C_{i}(t)$ of equation (4) is a function of $-x_{-i}(t)$ that treats increases and decreases of opponents' aggregate demands asymmetrically. One might instead choose to treat increases and decreases entirely symmetrically, replacing the term $\int p(t) d C_{i}(t)$ with the term $-\int p(t) d x_{-i}(t)$. At the same time, the term $C_{i}(t)$ of equation (4) implicitly incorporates a constant term in which the residual supply, $\mathbf{S}-x_{-i}(0)$, is priced at price $p(0)$ at time zero. Thus, one could define instead the payment, $a_{i}(T)$, of bidder $i$ by the following equation:

$$
a_{i}(T)=p(0)\left[\mathbf{S}-x_{-i}(0)\right]-\int_{0}^{T} p(t) d x_{-i}(t)
$$

By its construction, the principal difference between the original notion of "clinching" in equations (4)-(5) and the extended notion in equation (6) occurs when $x_{-i}(t)$ is nonmonotonic. In equations (4)-(5), price is integrated against $d C_{i}(t)$, so units are won when opponents' demands decrease, but units are not lost when opponents' demands correspondingly increase. By contrast, in equation (6), price is integrated against $d x_{-i}(t)$, so decreases and increases in opponents' demands are treated entirely symmetrically, allowing both "crediting" and "debiting" to occur.

An example of the calculation of the "crediting and debiting" Stieltjes integral of equation (6) (as well as an introduction to the vector notation that will be used for heterogeneous types of commodities) is provided by reexamining the example of Section I of the current article. Table 1 implies:

$$
d \mathbf{x}_{-1}(t)= \begin{cases}(-1,-1), & \text { if } t=1 \\ (-1,-2), & \text { if } t=2 \\ (-1,+1), & \text { if } t=3 \\ (-1,0), & \text { if } t=4 \\ (0,0), & \text { if } t \neq 1,2,3,4\end{cases}
$$


We need only note the value of the price vector $\mathbf{p}(t)$ at the times when $d \mathbf{x}_{-\mathbf{i}}(t) \neq 0$ :

$$
\mathbf{p}(t)= \begin{cases}(4,5), & \text { if } t=1 \\ (5,7), & \text { if } t=2, \\ (6,7), & \text { if } t=3 \\ (7,8), & \text { if } t=4\end{cases}
$$

Consequently, $-\int_{0}^{4} p^{1}(t) d x_{-1}^{1}(t)=-4 \times$ $(-1)-5 \times(-1)-6 \times(-1)-7 \times(-1)=$ 22 and $-\int_{0}^{4} p^{2}(t) d x_{-1}^{2}(t)=-5 \times(-1)-7 \times$ $(-2)-7 \times 1-8 \times 0=12$, or, in short, $-\int_{0}^{4} \mathbf{p}(t) \cdot d \mathbf{x}_{-1}(t)=22+12=34$, confirming the results summarized in the second column of Table 2 .

Nevertheless, the differences between the clinching approach and the crediting/debiting approach should not be overstated. Suppose that, in an ascending auction of homogeneous goods, we replace equations (4)-(5) with equation (6), and we choose $p(0)$ so that $x_{-i}(0)=S$. Then the central result from Ausubel (1997, 2004) continues to hold - sincere bidding by all bidders is an equilibrium-and the bidders' equilibrium payoffs remain unchanged. Thus, reformulating the payoffs need not disturb incentive compatibility.

\section{The Extension to $K$ Heterogeneous Commodities}

The most naïve way that one might think about generalizing the homogeneous goods procedure to the case of $K$ heterogeneous commodities is to run $K$ price clocks (one for each commodity) simultaneously, to compute the "credits" and "debits" for each independently, and to sum them up. Let the movement of the $K$ price clocks be described by a continuous, piecewise smooth, ${ }^{11}$ vector-valued function $\mathbf{p}(t)=\left(p^{1}(t), \ldots, p^{K}(t)\right)$ from $[0, T]$ to $\mathbb{R}^{K}$.

\footnotetext{
${ }^{11}$ The (vector-valued) continuous function $\mathbf{p}$ is said to be piecewise smooth if each coordinate $p^{k}$ has a bounded derivative which is continuous everywhere in $[0, T]$, except (possibly) at a finite number of points. At these exceptional points it is required that both right- and left-hand derivatives exist. A curve $\Gamma$ is said to be piecewise smooth if it can be described by a piecewise smooth function (Apostol, 1957, Definition 9-61).
}

Further suppose that each bidder $i$ bids according to the vector-valued function $\mathbf{x}_{\mathbf{i}}(t)=$ $\left(x_{i}^{1}(t), \ldots, x_{i}^{K}(t)\right)$ from $[0, T]$ to $X_{i}$, which is constrained to be of bounded variation in each coordinate $k$. Then the naïve extension of equation (6) would be to define $x_{-i}^{k}(t)=\sum_{j \neq i} x_{j}^{k}(t)$, for $k=1, \ldots, K$, and to define payments by:

$$
\begin{gathered}
a_{i}(T)=\mathbf{p}(0) \cdot\left[\mathbf{S}-\mathbf{x}_{-\mathbf{i}}(0)\right] \\
-\int_{0}^{T} \mathbf{p}(t) \cdot d \mathbf{x}_{-\mathbf{i}}(t) \\
\equiv \sum_{k=1}^{K}\left\{p^{k}(0)\left[S^{k}-x_{-i}^{k}(0)\right]-\int_{0}^{T} p^{k}(t) d x_{-i}^{k}(t)\right\}
\end{gathered}
$$

where the integrals of equation (7) are calculated as Stieltjes integrals. We begin by observing:

LEMMA 1: If $\mathbf{p}(\cdot)$ is continuous and if $x_{j}^{k}(\cdot)$ is of bounded variation for every bidder $j \neq i$ and commodity $k$, then the payment $a_{i}(T)$ of equation (7) is well defined.

The proofs of all lemmas and theorems appear in Appendix A.

Next, in order for the payment formula of equation (7) to serve its intended purpose, it is critical for us to establish the property of path independence. Suppose that bidders $j \neq i$ bid $\mathbf{q}_{\mathbf{j}}(\cdot)$, sincere bids relative to their utility functions. Consider two different price paths, $\mathbf{p}^{\mathbf{A}}(\cdot)$ and $\mathbf{p}^{\mathbf{B}}(\cdot)$, which originate at the same price vector and conclude at the same price vector. We need to show that the line integrals calculated along the two paths are equal, i.e., $\int_{0}^{T} \mathbf{p}^{\mathbf{A}}(t) \cdot d \mathbf{q}_{-\mathbf{i}}^{\mathbf{A}}(t)=\int_{0}^{T} \mathbf{p}^{\mathbf{B}}(t) \cdot d \mathbf{q}_{-\mathbf{i}}^{\mathbf{B}}(t)$. Otherwise, bidder $i$ would have the incentive to manipulate her demand reports so as to alter the price adjustment path to her advantage.

In general, path independence requires that the line integral along any (piecewise smooth) path from point $\mathrm{A}$ to point $\mathrm{B}$ gives the same value. Equivalently, a path is said to be closed if its starting and ending point are the same; path independence requires that the line integral along any (piecewise smooth) closed path equals zero. 
The classical consumer theory problem of integrability is the best-known problem in microeconomics in which the issue of path independence arises. There, the question considered is: What are necessary and sufficient conditions on a vector-valued function $\mathbf{x}_{\mathbf{i}}(\cdot)$ satisfying Walras's law to assure that it is the demand function derived from some utility function ${ }^{12}$ Recall that the answer is: $\mathbf{x}_{\mathbf{i}}(\cdot)$ must satisfy the symmetry condition, $\partial x_{i}^{j} / \partial p^{k}=\partial x_{i}^{k} / \partial p^{j}$. The reason is that, for path independence, $\mathbf{x}_{\mathbf{i}}(\cdot)$ needs to be the gradient of a potential function (i.e., there must exist a function $\phi_{i}(\cdot)$ such that $\nabla \phi_{i}(\cdot)=$ $\left.\mathbf{x}_{\mathbf{i}}(\cdot)\right)$. Furthermore, the symmetry condition on derivatives is essentially necessary and sufficient for the existence of such a potential function (e.g., see Apostol, 1957, Theorems 10-38, 10-45, and 10-48).

In the classical case of integrability, the potential function has the interpretation of an expenditure function. Path independence is the requirement that the incremental expenditure needed for an agent to attain a fixed level of utility, as the price vector changes from $\mathbf{p}^{\mathbf{A}}$ to $\mathbf{p}^{\mathbf{B}}$, must not depend on the particular price adjustment path taken from $\mathbf{p}^{\mathbf{A}}$ to $\mathbf{p}^{\mathbf{B}}$. Outside of economics, a better-known example of path independence is the analysis of a gravitational field in Newtonian mechanics. In a frictionless world, the amount of work required to move an object from point A to point $\mathrm{B}$ is the same along any possible path.

Thus, in the current auction context, path independence of the payment formula of equation (7) requires the existence of a potential function. For concise notation, we let $\overline{\mathbf{x}}_{-\mathbf{i}}(\mathbf{p}(t))=\left\{\mathbf{x}_{\mathbf{j}}(\mathbf{p}(t))\right\}_{j \neq i}$ denote the vector of demands by bidder $i$ 's opponents at time $t$, and we define $U_{-i}\left(\overline{\mathbf{x}}_{-\mathbf{i}}(\mathbf{p}(t))\right)=\sum_{j \neq i} U_{j}\left(\mathbf{x}_{\mathbf{j}}(\mathbf{p}(t))\right)$. With sincere bidding, the following important lemma shows that the potential function, $U_{-i}\left(\overline{\mathbf{x}}_{-\mathbf{i}}(\mathbf{p}(\cdot))\right)$, is associated with the crediting/ debiting formula, implying path independence. For greater generality, the result is obtained using subgradients rather than gradients, so that Lemma 2 applies to both the continuous and discrete models:

\footnotetext{
${ }^{12}$ In addition, the function $\mathbf{x}_{\mathbf{i}}(\cdot)$ is required to satisfy a negative semidefiniteness condition and the utility function is required to satisfy quasiconcavity, monotonicity, and continuity.
}

LEMMA 2: Suppose that $\mathbf{p}(\cdot)$ is a continuous, piecewise smooth function from $[0, T]$ to $\mathbb{R}^{K}$. Also suppose that $\mathbf{x}_{\mathbf{j}}(\cdot)$ is a measurable selection from $Q_{j}(\mathbf{p}(\cdot))$, the demand correspondence from a concave, continuous utility function $U_{j}(\cdot)$, and that $x_{j}^{k}(\cdot)$ is of bounded variation, for every bidder $j \neq i$ and commodity $k$. Then the integral $\int_{0}^{T} \mathbf{p}(t) \cdot d \mathbf{x}_{-\mathbf{i}}(\mathbf{p}(t))$ of equation (7) is independent of the path from $\mathbf{p}(0)$ to $\mathbf{p}(T)$ and equals:

$$
\text { (8) } \begin{aligned}
& \int_{0}^{T} \mathbf{p}(t) \cdot d \mathbf{x}_{-\mathbf{i}}(\mathbf{p}(t)) \\
& =U_{-i}\left(\overline{\mathbf{x}}_{-\mathbf{i}}(\mathbf{p}(T))\right)-U_{-i}\left(\overline{\mathbf{x}}_{-\mathbf{i}}(\mathbf{p}(0))\right) \\
& \equiv \sum_{j \neq i}\left[U_{j}\left(\mathbf{x}_{\mathbf{j}}(\mathbf{p}(T))\right)-U_{j}\left(\mathbf{x}_{\mathbf{j}}(\mathbf{p}(0))\right)\right] .
\end{aligned}
$$

\section{The Dynamic Auction Game for Divisible Commodities}

The auction is modeled as a dynamic game in continuous time. There are $n$ players. To simplify matters, we henceforth assume for the model of divisible commodities that utility functions, $U_{i}(\cdot)$, are strictly concave, making the sincere demand correspondences single-valued at all prices. At each time $t \in[0, \infty)$, a price vector $\mathbf{p}(t)$ is announced to the players. Each player $i$ then reports an optimal consumption bundle $\mathbf{x}_{\mathbf{i}}(t)$. The law of motion for the price vector is any continuous, sign-preserving transformation of the Walrasian tâtonnement price adjustment process (as formalized by Paul A. Samuelson, 1941):

Walrasian tâtonnement. Let $\mathbf{Z}(t)=-\mathbf{S}+\sum_{i=1}^{n}$ $\mathbf{x}_{\mathbf{i}}(t)$ denote the excess demand vector. Let $h(\cdot): \mathbb{R}^{K} \rightarrow \mathbb{R}^{K}$ denote any continuous function that is sign preserving in the sense that $h^{k}(\overrightarrow{\mathbf{z}})>0 \Leftrightarrow z^{k}>0$ and $h^{k}(\overrightarrow{\mathbf{z}})<0 \Leftrightarrow z^{k}<0$. Prices adjust according to:

(9) $\dot{p}^{k}(t)=h^{k}(\mathbf{Z}(t)), \quad$ for $k=1, \ldots, K$.

Given the initial price, $\mathbf{p}(0)$, and suitable restrictions on $\left\{\mathbf{x}_{\mathbf{i}}(s)\right\}_{i=1}^{n}$, equation (9) determines the evolution of the price vector, $\mathbf{p}(t)$, at all times $t \in[0, \infty)$. 
Let $H_{i}^{t}$ denote the part of the history of play prior to time $t$ that is observable to player $i$ at time $t$. One sensible specification is that $H_{i}^{t}$ comprises the history of aggregate excess demand and player $i$ 's own actions, i.e., $H_{i}^{t}=$ $\left\{\mathbf{Z}(s)\right.$ and $\left.\mathbf{x}_{\mathbf{i}}(s): s \in[0, t)\right\}$. Observe that, given equation (9), this observable history determines $\mathbf{p}(s)$ for all $s \in[0, t] .{ }^{13}$ The strategy $\sigma_{i}\left(t, H_{i}^{t}\right)$ of player $i(i=1, \ldots, n)$ is a function associating times and observable histories with elements of $X_{i}$. The strategy spaces $\Sigma_{i}$ may be any sets of functions $\sigma_{i}\left(t, H_{i}^{t}\right)$ which: (a) include sincere bidding; and (b) induce actions $\mathbf{x}_{\mathbf{i}}(t)$ by bidder $i$ that are piecewise continuous and of bounded variation, for each bidder $i$ and each commodity $k{ }^{14}$ The following theorems may be proven for many possible choices of strategy spaces; for specificity, we will use the following:

Piecewise Lipschitz-continuous functions. The strategy space of each player $i$ is given by: $\Sigma_{i}=\left\{\sigma_{i}:[0, \infty) \times \mathbb{R}^{K} \rightarrow X_{i}\right.$ such that $\sigma_{i}$ is a piecewise Lipschitz-continuous function of $(t, \mathbf{p})\}^{15}$

The strategies in $\Sigma_{i}$ are similar in spirit to "Markovian" strategies in the sense that a player has full knowledge of the history of aggregate excess demands (or even of individual demands), yet the player chooses to base her demands only on the current time and price. This strategy space is restrictive enough to induce actions satisfying the hypothesis of Lemma 2, while general enough to include sincere bidding.

The auction is said to terminate at time $T$ if

\footnotetext{
${ }^{13}$ Another sensible specification is that the observable history comprises the complete history of individual demands, i.e., $H_{i}^{t}=\left\{\mathbf{x}_{\mathbf{j}}(s): s \in[0, t)\right.$ and $\left.j=1, \ldots, n\right\}$.

${ }^{14}$ More precisely, we assume that for every player $i$, there exists a partitioning $0=t_{i}^{0}<t_{i}^{1}<\cdots<t_{i}^{l}<\cdots$ of the time interval $[0, \infty)$ by the points $t_{i}^{l}$ without finite points of accumulation, such that within each piece $\left[t_{i}^{l}, t_{i}^{l+1}\right)$ of the domain, the function $\mathbf{x}_{\mathbf{i}}(\cdot)$ is continuous, and each $x_{i}^{k}(\cdot)$ is required to be a function of bounded variation on every finite time interval $[0, T]$.

${ }^{15}$ More precisely, we assume that for every player $i$, there exists a partitioning $0=t_{i}^{0}<t_{i}^{1}<\cdots<t_{i}^{l}<\cdots$ of the time interval $[0, \infty)$ by the points $t_{i}^{l}$ without finite points of accumulation, and there exists a constant $C>0$, such that within each piece $\left[t_{i}^{l}, t_{i}^{l+1}\right) \times \mathbb{R}^{K}$ of the domain, the function $\mathbf{x}_{\mathbf{i}}(t, \mathbf{p})$ is Lipschitz-continuous in $(t, \mathbf{p})$, i.e., for $\left(t_{1}, \mathbf{p}_{\mathbf{1}}\right),\left(t_{2}\right.$, $\left.\mathbf{p}_{\mathbf{2}}\right) \in\left[t_{i}^{l}, t_{i}^{l+1}\right) \times \mathbb{R}^{K}$, we have $\left|x_{i}^{k}\left(t_{2}, \mathbf{p}_{2}\right)-x_{i}^{k}\left(t_{1}, \mathbf{p}_{\mathbf{1}}\right)\right| \leq$ $C\left(\left|t_{2}-t_{1}\right|+\left|\mathbf{p}_{2}-\mathbf{p}_{1}\right|\right)$.
}

$\sum_{i=1}^{n} \mathbf{x}_{\mathbf{i}}(T)=\mathbf{S}$, i.e., the aggregate demand equals the supply for every commodity. It is said to terminate at time $T=\infty$ if $\lim _{T \rightarrow \infty} \sum_{i=1}^{n}$ $\mathbf{x}_{\mathbf{i}}(T)=\mathbf{S}$. Following termination of the auction, players receive their quantities demanded at the termination time, $\mathbf{x}_{\mathbf{i}}(T)\left(\right.$ or $\left.\lim _{T \rightarrow \infty} \mathbf{x}_{\mathbf{i}}(T)\right)$, and payments are assessed according to equation (7). If the auction fails to terminate, i.e., if $\lim _{T \rightarrow \infty} \sum_{i=1}^{n} \mathbf{x}_{\mathbf{i}}(T) \neq \mathbf{S}$ or if $\lim _{T \rightarrow \infty} \sum_{i=1}^{n} \mathbf{x}_{\mathbf{i}}(T)$ fails to exist, then every player is assigned a payoff of $-\infty$.

The next lemma shows that, if all bidders bid sincerely, then starting from any history the auction converges to a Walrasian equilibrium price vector. The proof is little more than the classical argument (see, for example, Hal R. Varian, 1981, pp. 104-06) that, with pricetaking agents, the price is globally convergent to a Walrasian equilibrium price vector. We have:

LEMMA 3: With divisible goods and strictly concave utility functions for all bidders, and after any history, sincere bidding by every bidder $i$ induces convergence to a Walrasian equilibrium price vector.

The information structure of the auction game may be one of complete or incomplete information regarding opposing bidders' valuations. With complete information, each bidder is fully informed of the functions $\left\{U_{j}(\cdot)\right\}_{j=1}^{n}$, and the appropriate equilibrium concept is subgame perfect equilibrium. With incomplete information, each bidder $i$ is informed only of her own utility function $U_{i}(\cdot)$ and of the joint probability distribution $F(\cdot)$ from which the profile $\left\{U_{j}(\cdot)\right\}_{j=1}^{n}$ is drawn. In static games of incomplete information, authors sometimes advocate ex post equilibrium, which requires that the strategy for each player would remain optimal if the player were to learn her opponents' types (see Jacques Crémer and Richard P. McLean, 1985). In the current dynamic game, the equilibrium concept that we will use (which we defined and also used in Ausubel, 2004) is ex post perfect equilibrium, which imposes this same condition at every node of the auction game:

Ex post perfect equilibrium. The strategy $n$ tuple $\left\{\sigma_{i}\right\}_{i=1}^{n}$ is said to comprise an ex post perfect equilibrium if for every time $t$, fol- 
lowing any history $H_{i}^{t}$, and for every realization $\left\{U_{i}\right\}_{i=1}^{n}$ of private information, the $n$-tuple of continuation strategies $\left\{\sigma_{i}(\cdot, \cdot \mid t\right.$, $\left.\left.H_{i}^{t}, U_{i}\right)\right\}_{i=1}^{n}$ constitutes a Nash equilibrium of the game in which the realization of $\left\{U_{i}\right\}_{i=1}^{n}$ is common knowledge.

Alternatively, we could have explicitly defined beliefs for each bidder and stated the theorems of this article in terms of the perfect Bayesian equilibrium concept. ${ }^{16}$ Stating the results in their current form, however, gives them a number of additional desirable properties, e.g., the results are independent of the underlying distribution of bidders' types (see Crémer and McLean, 1985; Maskin, 1992; Perry and Reny, 2002, 2005). The results as stated also encompass the complete-information version of the model, since ex post perfect equilibrium then reduces to the familiar equilibrium concept of subgame perfect equilibrium.

Our assumptions above, which assure that the strategies $\sigma_{i} \in \Sigma_{i}$ induce demands $\mathbf{x}_{\mathbf{i}}(t)$ by each bidder $i$ that are piecewise continuous and of bounded variation in each coordinate $k$, and that the price adjustment process $\dot{\mathbf{p}}(t)$ is continuous in $\mathbf{x}_{\mathbf{i}}(t)$, guarantee that the technical requirements for Lemma 2 hold. In light of the path independence established by Lemma 2, if the bidders $j \neq i$ bid sincerely relative to strictly concave utility functions, then the strategic choice by bidder $i$ reduces from an optimization problem over price paths in $\mathbb{R}^{K}$ to one over endpoints in $\mathbb{R}^{K}$, implying:

THEOREM 1: With divisible goods, if each opposing bidder $j \neq i$ bids $\left.\mathbf{q}_{\mathbf{j}} \mathbf{( p}(\cdot)\right)$, a sincere bid relative to a strictly concave utility function $U_{j}(\cdot)$, then bidder $i$ with strictly concave utility function $U_{i}(\cdot)$ maximizes her payoff by bidding sincerely. By bidding sincerely, bidder i selects

\footnotetext{
${ }^{16}$ To state the results in terms of perfect Bayesian equilibrium, we would begin by specifying that, after any history, each player $i$ has posterior beliefs over opponents' utility functions, $U_{-i}(\cdot) \equiv\left\{U_{j}(\cdot)\right\}_{j \neq i}$. The beliefs of player $i$ are denoted $\mu_{i}\left(\cdot \mid t, H_{i}^{t}, U_{i}\right)$. The $n$-tuple $\left\{\sigma_{i}, \mu_{i}\right\}_{i=1}^{n}$ is then defined to comprise a perfect Bayesian equilibrium if the strategies $\sigma_{i} \in \Sigma_{i}$, the beliefs $\mu_{i}$ are updated by Bayes's rule whenever possible, and if following any history $H^{t}$ of play prior to time $t, \sigma_{i}$ is a best response for player $i$ in the continuation game against $\left\{\sigma_{j}\right\}_{j \neq i}$ given beliefs $\mu_{i}\left(\cdot \mid t, H_{i}^{t}\right.$, $\left.U_{i}\right)$, and for every $i=1, \ldots, n$.
}

a Walrasian equilibrium price vector as the endpoint of the price path and maximizes social surplus, $U_{i}(\cdot)+\sum_{j \neq i} U_{j}(\cdot)$, over all feasible allocations. This result holds at every time $t$ and after every history $H_{i}^{t}$.

This theorem is established by using Lemma 2 to show that the portion of payoff that a bidder is able to influence coincides with the social surplus associated with the allocation implied by the terminal price vector, $\mathbf{p}(T)$. Consequently, the bidder's payoff is maximized if and only if $\mathbf{p}(T)$ is a Walrasian equilibrium price vector. By Lemma 3, bidder $i$ can attain a Walrasian equilibrium by bidding sincerely.

Theorem 1 immediately implies that sincere bidding is an ex post perfect equilibrium of the new auction game. We have:

THEOREM 2: With divisible goods, strictly concave utility functions, mandatory participation, and any arbitrary initial price vector of $\mathbf{p}(0)$ :

(a) Sincere bidding by every bidder is an ex post perfect equilibrium of the auction game;

(b) With sincere bidding, the price vector converges to a Walrasian equilibrium price vector; and

(c) With sincere bidding, the outcome is that of the modified VCG mechanism with price of $\mathbf{p}(0)$.

Note that the hypothesis of Theorem 2 assumes "mandatory participation." This refers to the fact that bidders' payments depend on the initial price vector $\mathbf{p}(0)$ (see equation (7)) and it is entirely possible, with some initial price vectors, that sincere bidding may yield a negative payoff (see also footnote 5). For example, when $\mathbf{p}(0)$ exceeds the Walrasian equilibrium price vectors, the payment of bidder $i$ for receiving $\mathbf{x}_{\mathbf{i}}^{*}$ might exceed $U_{i}\left(\mathbf{x}_{i}^{*}\right)$. In that event, if given a choice whether or not to participate, bidder $i$ with complete information would choose to stay out of the auction. We would be assured of the conclusion of Theorem 2 only if bidders' participation was mandatory.

Mandatory participation ceases to be an issue, however, if the auction procedure is en- 
hanced in such a way that bidders receive their VCG payoffs. The VCG mechanism is ex post individually rational (i.e., the bidder's payoff is always nonnegative and so, under voluntary participation, the bidder would choose to participate). We turn to developing such an enhancement to the auction procedure in the next section.

\section{Relationship with the Vickrey-Clarke- Groves Mechanism}

In Theorem 2, each bidder $i$ received her payoff from the modified VCG mechanism with price of $\mathbf{p}(0)$. This payoff coincides with bidder $i$ 's VCG payoff if the initial price vector $\mathbf{p}(0)$ is chosen appropriately. Moreover, since the VCG payoff is nonnegative, we no longer need to assume mandatory participation. We have:

THEOREM 3: With divisible goods and strictly concave utility functions, if the initial price $\mathbf{p}(0)$ is chosen such that the market without bidder $i$ clears at $\mathbf{p}(0)$ (i.e., $\sum_{j \neq i} \mathbf{q}_{\mathbf{j}}(\mathbf{p}(0))=\mathbf{S}$ ) and if each bidder $j \neq i$ bids sincerely, then bidder $i$ maximizes her payoff by bidding sincerely and thereby receives her VCG payoff.

Furthermore, the hypothesis of Theorem 3 is trivial to satisfy if all bidders are identical, providing a simple procedure to generate the VCG outcome. We immediately have the following corollary:

COROLLARY TO THEOREM 3: With identical bidders, divisible goods and strictly concave utility functions, if the initial price $\mathbf{p}(0)$ is chosen such that the market without one bidder clears (i.e., if $\left.\sum_{j \neq i} \mathbf{q}_{\mathbf{j}}(\mathbf{p}(0))=\mathbf{S}\right)$, then the sincere bidding equilibrium of the auction game gives the same outcome as the VCG mechanism.

Without identical bidders, however, it obviously is not generally possible to select an initial price $\mathbf{p}(0)$ such that every bidder receives her VCG payoff. Theorem 3 nevertheless suggests a more intricate, parallel auction procedure that could be followed so that every bidder receives exactly her VCG payoff.

Parallel Auction Game.-Begin with any initial price $\mathbf{p}(0) \in \mathbb{R}^{K}$. First, we perform the following $n$ steps, which may be done in any order or may be run in parallel:

Step 1: Run the auction procedure of announcing a price $\mathbf{p}(t)$, allowing each bidder $i$ to respond with a quantity $\mathbf{x}_{\mathbf{i}}(t)$, and adjusting price according to $\dot{\mathbf{p}}(t)=-\mathbf{S}+\sum_{i \neq 1} \mathbf{x}_{\mathbf{i}}(t)$, starting from price $\mathbf{p}(0)$ and until a price $\mathbf{p}_{-\mathbf{1}}$ is determined at which the market (absent bidder 1) clears.

...

Step $n$ : Run the auction procedure of announcing a price $\mathbf{p}(t)$, allowing each bidder $i$ to respond with a quantity $\mathbf{x}_{\mathbf{i}}(t)$, and adjusting price according to $\dot{\mathbf{p}}(t)=-\mathbf{S}+\sum_{i \neq n} \mathbf{x}_{\mathbf{i}}(t)$, starting from price $\mathbf{p}(0)$ and until a price $\mathbf{p}_{-\mathbf{n}}$ is determined at which the market (absent bidder $n$ ) clears.

Second, we perform the following additional $n$ steps, which again may be done in any order or may be run in parallel, which are similar to the first $n$ steps except that all $n$ bidders' demands are now included in the Walrasian tâtonnement process:

Step $n+1$ : Run the auction procedure of announcing a price $\mathbf{p}(t)$, allowing each bidder $i$ to respond with a quantity $\mathbf{x}_{\mathbf{i}}(t)$, and adjusting price according to equation (9), starting from price $\mathbf{p}_{-\mathbf{1}}$ and continuing until a price $\mathbf{p}(T)$ is determined at which the market (with all bidders included) clears.

Step $2 n$ : Run the auction procedure of announcing a price $\mathbf{p}(t)$, allowing each bidder $i$ to respond with a quantity $\mathbf{x}_{\mathbf{i}}(t)$, and adjusting price according to equation (9), starting from price $\mathbf{p}_{-\mathbf{n}}$ and continuing until a price $\mathbf{p}(T)$ is determined at which the market (with all bidders included) clears.

Finally, payoffs are computed as follows. The commodity bundle assigned to each bidder $i(1 \leq$ $i \leq n)$ is given by $\mathbf{x}_{\mathbf{i}}(T)$ from step $(n+i)$ above. The payment is given by the line integral of equation (7), calculated from step $(n+i)$ above, i.e., along the path from $\mathbf{p}_{-\mathbf{i}}$ to $\mathbf{p}(T)$. If the results of steps $n+1, \ldots, 2 n$ are inconsistent (i.e., if they yield different allocations), then every bidder is assigned a payoff of $-\infty$.

The parallel auction procedure is illustrated in Figure 1. As the figure makes clear, a number 


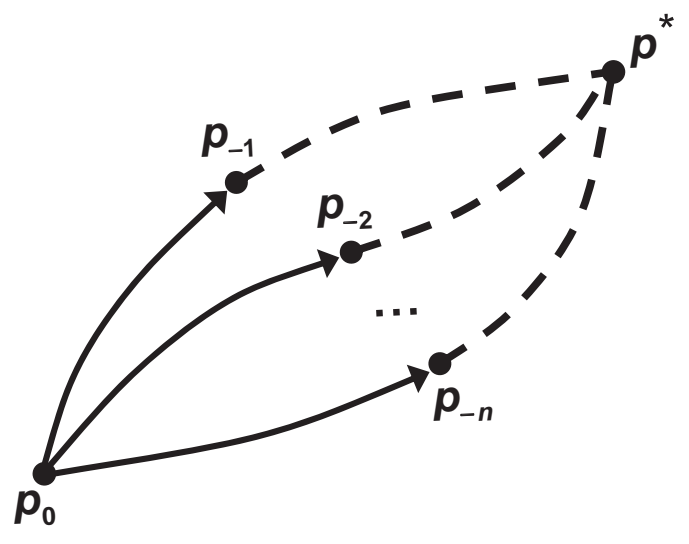

Figure 1. Parallel Auction Game

of the steps described above are unnecessary. Observe that one could delete all but one of the dashed arcs, yet all of the nodes in the figure would remain connected. Hence, by the path independence of Lemma 2, one would still have sufficient information to calculate all of the needed line integrals. Moreover, if one deletes $(n-1)$ of the last $n$ steps, one is able to get rid of the awkward final step of checking whether the results of steps $n+1, \ldots, 2 n$ are consistent, and assigning each bidder a payoff of $-\infty$ if they are inconsistent. Thus, in performing the parallel auction, we may utilize the following shortcut procedure:

Shortcut Procedure for a Parallel Auction. Only $(n+1)$ of the steps described above-all of the first $n$ steps, but only one of the last $n$ steps - actually need to be carried out.

For example, suppose that out of the last $n$ steps, only Step $2 n$ is performed. Payoffs can still be computed as follows. The payment of bidder $n$ is given by the line integral of equation (7), calculated along the path from $\mathbf{p}_{-\mathbf{n}}$ to $\mathbf{p}^{*}$, i.e., by step $2 n$ alone. The payment of bidder $i$ $(1 \leq i \leq n-1)$ is also given by equation (7), but is calculated as a line integral along the union of three paths: the path from $\mathbf{p}_{-\mathbf{i}}$ to $\mathbf{p}(0)$; the path from $\mathbf{p}(0)$ to $\mathbf{p}_{-\mathbf{n}}$; and the path from $\mathbf{p}_{-\mathbf{n}}$ to $\mathbf{p}^{*}$. Step $i$ generated the first path (run backward), as well as all necessary demands for calculating equation (7); step $n$ generated the second path; and step $2 n$ generated the third path. For both the full parallel auction game and for the shortcut procedure, we have:
THEOREM 4: With divisible goods, strictly concave utility functions, and any initial price vector, sincere bidding by every bidder is an ex post perfect equilibrium of the parallel auction game, prices converge to a Walrasian equilibrium price vector, and the outcome is exactly that of the VCG mechanism.

\section{The Dynamic Auction Game for Discrete Goods}

We now turn to an environment of indivisible goods. A seller wishes to allocate units of each of $K$ types of discrete heterogeneous commodities among $n$ bidders. The seller's available supply of commodities is denoted by $\mathbf{S}=\left(S^{1}\right.$, $\left.\ldots, S^{K}\right) \in \mathbb{Z}_{++}^{K}$. Bidder $i$ 's consumption set is the set $X_{i}=\left\{\mathbf{x}_{\mathbf{i}} \in \mathbb{Z}^{K}: 0 \leq x_{i}^{k} \leq z_{i}^{k}\right.$ for all $k=$ $1, \ldots, K\}$ bounded below by zero and bounded above by the vector $\mathbf{z}_{\mathbf{i}}=\left(z_{i}^{1}, \ldots, z_{i}^{K}\right) \in \mathbb{Z}_{+}^{K}$. Bidder $i$ 's consumption bundle is denoted by $\mathbf{x}_{\mathbf{i}}$ $=\left(x_{i}^{1}, \ldots, x_{i}^{K}\right) \in X_{i}$.

In order to treat the case of discrete commodities, it will now be necessary for us to impose the substitutes condition. This condition, often known as "gross substitutes,"17 requires that if the prices of some commodities are increased while the prices of the remaining commodities are held constant, then a bidder's sincere demand weakly increases for each of the commodities whose prices were held constant. The reason for requiring the substitutes condition in the discrete case is to assure the existence of Walrasian equilibrium. ${ }^{18}$ We define:

DEFINITION 4: Consider an economy with $K$ indivisible commodities, each of which is available in a supply of one. $U_{i}(\cdot)$ is said to satisfy the substitutes condition if, for any two price

\footnotetext{
${ }^{17}$ The substitutes condition is often referred to as gross substitutes (as opposed to net substitutes, which would be the case if the analogous condition held for compensated demands). In the current context of quasilinear utility, however, there is no distinction between gross substitutes and net substitutes, so in this article, the condition will simply be called the substitutes condition.

${ }^{18}$ Indeed, in the case of discrete items, given any one bidder with preferences violating the substitutes condition, it is possible to specify another bidder with additive preferences and an endowment of goods such that the resulting economy has no Walrasian equilibrium (Milgrom, 2000, Theorem 4).
} 
vectors $\mathbf{p}$ and $\mathbf{p}^{\prime}$ such that $\mathbf{p}^{\prime} \geq \mathbf{p}$ and demand $\mathbf{q}_{\mathbf{i}}(\cdot)$ is single valued at $\mathbf{p}$ and $\mathbf{p}^{\prime}, q_{i}^{k}\left(\mathbf{p}^{\prime}\right) \geq q_{i}^{k}(\mathbf{p})$ for any commodity $k(1 \leq k \leq K)$ such that $p^{\prime k}=p^{k}$.

The assumption in Definition 4 that each commodity is available in a supply of one is without loss of generality, since if there are multiple units of some commodities, one can expand the commodity space by treating each unit of a commodity as a unique item (Bikhchandani and Mamer, 1997, sect. 2). The substitutes condition is defined with respect to this "unique items" formulation, since it is then a "sufficient and almost necessary" condition for the existence of Walrasian equilibrium. ${ }^{19}$ However, the auction procedure itself will be specified to allow multiple units of each discrete commodity. This provides a more compact way for bidders to communicate information, and is more naturally connected with both the traditional Walrasian auctioneer procedure and the divisible commodities treatment, above.

The substitutes condition is stronger than the concave utility assumption that we made above. Substitutes preferences imply concave utility. Let $u_{i}: \mathbb{R} \rightarrow \mathbb{R}$ be increasing and concave, however, and consider the utility function $U_{i}\left(\mathbf{x}_{\mathbf{i}}\right)=u_{i}\left(\min _{k=1, \ldots, K} x_{i}^{k}\right)$. Then $U_{i}(\cdot)$ is a concave utility function, but starting from a consumption vector $\mathbf{x}_{\mathbf{i}}=(\alpha, \ldots, \alpha)$, the various commodities are complements for bidder $i$. The following assumptions are made for the discrete case:

(A1') Integer pure private values: The utility function $U_{i}: X_{i} \rightarrow \mathbb{Z}$ takes integer values.

(A2') Quasilinearity: The same as (A2).

(A3') Monotonicity: The utility function $U_{i}$ : $X_{i} \rightarrow \mathbb{Z}$ is increasing, i.e., if $\mathbf{x}_{\mathbf{i}}^{\prime} \geq \mathbf{x}_{\mathbf{i}}$ and $\mathbf{x}_{\mathbf{i}}^{\prime} \neq \mathbf{x}_{\mathbf{i}}$, then $U\left(\mathbf{x}_{\mathbf{i}}^{\prime}\right)>U\left(\mathbf{x}_{\mathbf{i}}\right)$.

(A4') Substitutes condition: The utility function $U_{i}: X_{i} \rightarrow \mathbb{Z}$ satisfies Definition 4.

As before, the price vector will be denoted by $\mathbf{p}=\left(p^{1}, \ldots, p^{K}\right) \in \mathbb{R}^{K}$, but it will soon be

\footnotetext{
${ }^{19}$ Kelso and Crawford (1982), Gul and Stacchetti (1999), and Milgrom (2000).
}

specified to take nonnegative integer values at integer times. The demand correspondence, $Q_{i}(\mathbf{p})$, is defined as before.

The auction is modeled as a dynamic game. At each time $t=0,1,2, \ldots$, the price vector $\mathbf{p}(t)$ is announced to the $n$ players. Each player $i$ responds by reporting her set $\mathbf{x}_{\mathbf{i}}(t) \subset X_{i}$ of one or more optimal consumption bundles. The law of motion for the price variable will be specified later in this section.

Let $H_{i}^{t}$ denote the part of the history of play prior to time $t$ that is observable to player $i$ at time $t$. One sensible possibility is that the observable history comprises the complete history of price vectors and demand profiles, i.e., $H^{t}=$ $\left\{\mathbf{p}(s)\right.$ and $\mathbf{x}_{\mathbf{j}}(s): 0 \leq s<t$ and $\left.j=1, \ldots, n\right\}$. The strategy $\sigma_{i}\left(t, H^{t}\right)$ of a player $i(i=1, \ldots, n)$ is a set-valued function of times and observable histories $\sigma_{i}: \mathbb{Z}_{+} \times H_{i}^{t} \rightarrow 2^{X_{i}}$. The strategy space $\Sigma_{i}$ is the set of all such functions $\sigma_{i}\left(t, H_{i}^{t}\right)$. As in the divisible goods game of Sections V and VI, the equilibrium concept will be ex post perfect equilibrium. In the equilibrium that we construct, bidders will engage in sincere bidding, which now means that they report truthfully their entire demand correspondences. This sincere bidding strategy will simply be denoted by $Q_{i}(\mathbf{p})$, the same notation as for the demand correspondence.

The logic behind classical Walrasian tâtonnement remains sound in a discrete environment. However, the classic Walrasian tâtonnement process of differential equation (9), in which the price for each good is continuously increased (or decreased) in relation to excess demand (or supply) based on reports of demand functions by bidders, encounters major technical difficulties, as shown by the following example. Suppose that there are two indivisible goods, each available in a quantity of one, and three bidders, each with utility functions given by: $U(0,0)=$ $0 ; U(1,0)=c ; U(0,1)=c$; and $U(1,1)=c$. At a price vector of $(p, p)$, where $0<p<c$, each bidder would demand one of the goods, and so one good is in excess demand. Without loss of generality, say it is the first good, so its price is increased according to equation (9). At any price vector of ( $p+\varepsilon, p)$, however, where $\varepsilon>$ 0 , the aggregate demand for the first good drops to zero and for the second good jumps to three. Equation (9) now requires, instead, the price of the second good to rise. The choice of good 
whose price increases may oscillate back and forth arbitrarily frequently.

Gul and Stacchetti (2000) provide an elegant procedure that circumvents this difficulty and thereby extends classical Walrasian tâtonnement to environments with discrete goods and substitutes preferences. The solution that they give to the conundrum of the previous paragraph is to increase the prices of the two goods simultaneously; knowing to do this requires having the bidders report information about their demand correspondences (which indeed is how we have specified the game in this section). If agents bid sincerely by reporting all of their optimal commodity bundles at each price, the procedure is guaranteed to converge in finitely many steps from an initial price vector of zero to the lowest Walrasian equilibrium price vector, and to get there via an ascending price path. While the Gul-Stacchetti procedure remains subject to the same critique as classical Walrasian tâtonnement - that if bidders possess any market power then the posited bidder behavior fails to be incentive compatible (see the introduction)-it provides an extremely useful extension of Walrasian tâtonnement to environments with discrete goods.

Appendix B, summarizing the results of a companion paper (Ausubel, 2005), describes in detail a simplified version of Gul and Stacchetti's price adjustment procedure. It draws heavily from Gul and Stacchetti's work, but builds upon it to yield an improved procedure for the problem at hand. ${ }^{20}$ At the heart of the simplified

\footnotetext{
${ }^{20}$ The procedure developed in the companion paper and outlined in Appendix B departs from Gul and Stacchetti's (2000) procedure in several respects. First, the description and proof in the companion paper do not require any knowledge of matroid theory and instead rely on the (better known and more elementary) properties of submodular functions. Second, whereas Gul and Stacchetti define only an ascending price adjustment procedure, we utilize here both an ascending algorithm and a "global" algorithm (which may ascend or descend). While the ascending algorithm is guaranteed to converge to a Walrasian equilibrium price vector only starting from an initial price vector of zero, the global algorithm is guaranteed to converge from any initial price vector (i.e., global convergence). Third, whereas Gul and Stacchetti define their procedure for the "unique items" formulation, the current procedure is specified for the useful generalization where there may be multiple units of each "type" of commodity. Fourth, in step 2 of their procedure, Gul and Stacchetti (2000, p. 78) find it necessary to require
}

price adjustment procedure is a Lyapunov function-a construction familiar from the older literature on the global stability of price adjustment processes. In the global stability literature, given a dynamical system $\dot{\mathbf{p}}=f(\mathbf{p})$ with equilibrium $\mathbf{p}^{*}$, a Lyapunov function is defined to be a differentiable function $L(\mathbf{p})$ that exhibits two key properties: (a) $L(\cdot)$ is minimized at $\mathbf{p}^{*}$; and (b) $\dot{L}(\mathbf{p}(t))<0$ for all $\mathbf{p}(t) \neq \mathbf{p}^{*}$. The basic result is that, if there exists a Lyapunov function for the dynamical system, then $\mathbf{p}^{*}$ is a globally stable equilibrium (i.e., $\mathbf{p}(t) \rightarrow \mathbf{p}^{*}$ for every initial condition $\mathbf{p}(0))$. See, for example, Arrow and Hahn (1971, chap. 11) and Varian (1981).

In the classical derivation of global stability for Walrasian tâtonnement, when there are divisible commodities and consumers with quasilinear utility functions, the following Lyapunov function is used:

$$
L(\mathbf{p})=\mathbf{p} \cdot \mathbf{S}+\sum_{i=1}^{n} V_{i}(\mathbf{p}) .
$$

The reason that this function is selected in the divisible commodities formulation is that its subgradient at $\mathbf{p}$ equals $\mathbf{S}-\sum_{i=1}^{n} \mathbf{q}_{\mathbf{i}}(\mathbf{p})$, the excess supply vector (see the proof of Lemma 3 in Appendix A or such sources as Varian, 1981, pp. 104-06).

The companion paper establishes that the exact same Lyapunov function as in equation (10) can be successfully used in constructing a simplified and improved version of Gul and Stacchetti's tâtonnement algorithm for discrete goods. The minimizers of $L(\cdot)$ correspond to Walrasian equilibrium price vectors (Proposition 1), and the price adjustment process (now discrete) gives us a next price vector $\mathbf{p}(t+1)$ such that $L(\mathbf{p}(t+1))<L(\mathbf{p}(t))$ whenever $\mathbf{p}(t)$ is

\footnotetext{
the auction to end without trade if bidders ever report (untruthfully) in such a way that the excess demand set is not well defined. By contrast, the current procedure operates by making use of any minimal minimizing set-which always exists-so there is never a need for such a punishment. Fifth, the current procedure is specified in such a way as to yield constant demand profiles at times $\hat{t} \in[t, t+1)$, where $t$ is any integer. This guarantees that the line integral of equation (7) is well defined and so the current algorithm can be used as an input into our analysis of Sections V and VI.
} 
not a Walrasian equilibrium price vector. Appendix B summarizes the key steps in the argument. The reader is referred to the companion paper for the complete proofs of Propositions 1-5.

Given Definitions 5-7 and Propositions 1-5 in Appendix B, we may provide a complete description of an ascending tâtonnement algorithm for discrete goods:

(a) The auctioneer initializes $\mathbf{p}(0)=\mathbf{0}$ (or any initial price vector less than or equal to $\mathbf{p}$ ) and, at each $t=0,1,2, \ldots$, the auctioneer asks each bidder $i$ for a report, $Q_{i}(\mathbf{p}(t))$, of her demand correspondence at $\mathbf{p}(t)$.

(b) The auctioneer extends each bidder's demand correspondence report using Definition 5 and thereby determines indirect utility functions on the entire unit $K$-dimensional cube, $\{\mathbf{p}(t)+\boldsymbol{\Delta}: \mathbf{0} \leq \boldsymbol{\Delta} \leq \mathbf{1}\}$. If bidders have substitutes preferences and make truthful demand correspondence reports, the extended indirect utility functions are correct (Proposition 2).

(c) The auctioneer then determines a next price vector, $\mathbf{p}(t+1)$, satisfying Definition 7 , i.e., a minimal minimizer of the Lyapunov function $L(\cdot)$ of equation (10) on the vertices of $\{\mathbf{p}(t)+\boldsymbol{\Delta}: \mathbf{0} \leq \boldsymbol{\Delta} \leq \mathbf{1}\}$. Given substitutes preferences and truthful reporting, this minimal minimizer is unique and coincides with the unique minimal minimizer of the Lyapunov function $L(\cdot)$ on the entire unit $K$-dimensional cube, $\{\mathbf{p}(t)+\boldsymbol{\Delta}: \mathbf{0} \leq \boldsymbol{\Delta} \leq$ 1) (Proposition 3); but in the event of untruthful reporting, any minimal minimizer can be selected.

(d) The algorithm continues so long as $\mathbf{p}(t+$ 1) $\neq \mathbf{p}(t)$ and terminates when $\mathbf{p}(t+1)=$ $\mathbf{p}(t)$. Propositions 4 and 5 show that, given substitutes preferences and truthful reporting, the algorithm must terminate at $\mathbf{p}$. By Proposition 1, there exists an allocation $\left(\mathbf{x}_{\mathbf{1}}^{*}, \ldots, \mathbf{x}_{\mathbf{n}}^{*}\right)$ such that $\mathbf{x}_{\mathbf{i}}^{*} \in Q_{i}(\mathbf{p})$, for every $i=1, \ldots, n$, and the bidders may be assigned any such Pareto optimal allocation.

The ascending algorithm can serve as a replacement for the algorithm of Gul and Stacchetti (2000). It has the advantages described in footnote 20, but it has the limitation that it achieves convergence to a Walrasian equilibrium price vector only if the initial price vector $\mathbf{p}(0) \leq \overline{\mathbf{p}}$. The companion paper also provides a complete description of a descending tâtonnement algorithm for discrete goods. It is thoroughly analogous to the ascending algorithm, with the main differences that the auctioneer determines a next price vector, $\mathbf{p}(t+1)$, which is a maximal minimizer of the Lyapunov function $L(\cdot)$ of equation (10) on the vertices of $\{\mathbf{p}(t)-\boldsymbol{\Delta}: \mathbf{0} \leq \boldsymbol{\Delta} \leq \mathbf{1}\}$. Starting from an initial price vector $\mathbf{p}(0) \geq \overline{\mathbf{p}}$, the descending algorithm must terminate at $\overline{\mathbf{p}}$.

Finally, by combining the ascending and descending tâtonnement algorithms, the companion paper defines a global Walrasian tâtonnement algorithm for discrete goods:

(a) The auctioneer selects any initial price vector, $\mathbf{p}(0)$, and, at each $t=0,1,2, \ldots$, the auctioneer runs the ascending tâtonnement algorithm until it terminates at $\mathbf{p}\left(T_{1}\right)$.

(b) Starting from the price vector, $\mathbf{p}\left(T_{1}\right)$, where the ascending tâtonnement algorithm terminated, and, at each $t=T_{1}+1, T_{1}+2, \ldots$, the auctioneer runs the descending tâtonnement algorithm until it terminates at $\mathbf{p}\left(T_{2}\right)$.

(c) Starting from the price vector, $\mathbf{p}\left(T_{2}\right)$, where the descending tâtonnement algorithm terminated, and, at each $t=T_{2}+1, T_{2}+$ $2, \ldots$, the auctioneer runs the ascending tâtonnement algorithm until it terminates at $\mathbf{p}\left(T_{3}\right)$.

(d) The algorithm continues so long as either the ascending or descending tâtonnement algorithm has not terminated, and terminates at $\mathbf{p}(T)$ such that $\mathbf{p}(T+1)=\mathbf{p}(T)$ for both the ascending and descending tâtonnement algorithms.

The global Walrasian tâtonnement algorithm satisfies a global convergence theorem (see the companion paper): starting from any integervalued initial price vector and given truthful reporting by bidders, it converges to a Walrasian equilibrium price vector in finitely many iterations. Using the global Walrasian tâtonnement algorithm in place of the differential equation (9) classically used to describe Walrasian tâtonnement, we may extend the results of Sections V and VI for the continuous model and obtain analogous results for the discrete model.

Recall that the price paths and the payoff calculations of Sections V and VI were speci- 
fied in continuous time $t \in[0, \infty)$. Thus, it is convenient for us to convert the discrete price adjustment process for discrete goods into a continuous-time adjustment process. This can be done easily as follows. At each time $t=0,1$, $2, \ldots$, the auctioneer announces an integervalued price vector $\mathbf{p}(t)$, and bidders respond by reporting demand correspondences $\left\{\mathbf{x}_{\mathbf{i}}(t)\right\}_{i=1}^{n}$. If, in the iteration at time $t$, the global Walrasian tâtonnement algorithm is ascending and determines a set $E_{+}$of commodities associated with a minimal minimizer, the auctioneer can continuously (indeed, linearly) increase the price vector at times $\hat{t} \in[t, t+1)$ by:

$$
\dot{p}^{k}(\hat{t})= \begin{cases}1, & \text { if } k \in E_{+} \\ 0, & \text { if } k \notin E_{+}\end{cases}
$$

and if, in the iteration at time $t$, the global Walrasian tâtonnement algorithm is descending and determines a set $E_{-}$of commodities associated with a maximal minimizer, the auctioneer can continuously (indeed, linearly) decrease the price vector at times $\hat{t} \in[t, t+1)$ by:

$$
\dot{p}^{k}(\hat{t})= \begin{cases}-1, & \text { if } k \in E_{-} \\ 0, & \text { if } k \notin E_{-} .\end{cases}
$$

Given the continuous-time adjustment process specified by equations (11)-(12), observe that the payoff equation (7) and Lemmas 1 and 2 continue to hold literally as before. Thus, using essentially the same arguments as in Section V and VI, we have the following results for discrete goods:

THEOREM 1': With discrete goods and current price vector $\mathbf{p}$, if each opposing bidder $j \neq$ $i$ bids sincerely relative to a substitutes utility function $U_{j}(\cdot)$, then bidder $i$ with substitutes utility function $U_{i}(\cdot)$ maximizes her payoff by bidding sincerely, which has the effect of maximizing social surplus, $U_{i}(\cdot)+\sum_{j \neq i} U_{j}(\cdot)$, over all allocations.

The proof of Theorem 1' is similar to the proof of Theorem 1; the only novelty occurs in two places. First, the price adjustment procedure specified by equations (11)-(12) yields a continuous, piecewise smooth price path; in- deed, the price path is continuous and piecewise linear. Second, Proposition 2 in Appendix B assures us that, despite receiving demand reports from bidders at only integer times, the auctioneer can nevertheless reconstruct a constant profile of optimal demands for all of the bidders on each time interval $[t, t+1)$, where $t$ is an integer, and so each bidder's demand is of bounded variation. Thus, Lemmas 1 and 2 continue to hold. In particular, equation (7) continues to be well defined and path independent, and it can still be evaluated using equation (8).

This result then implies:

THEOREM 2': With discrete goods, the substitutes condition, and mandatory participation:

(a) Sincere bidding by every bidder is an ex post perfect equilibrium of the auction game;

(b) With sincere bidding and any arbitrary integer-valued initial price vector $\mathbf{p}(0)$, prices converge to a Walrasian equilibrium price vector in finite time; and

(c) With sincere bidding and any arbitrary integer-valued initial price vector $\mathbf{p}(0)$, the outcome is that of the modified VCG mechanism with price of $\mathbf{p}(0)$.

Analogous to the results in Section VI, we also have:

THEOREM 3': With discrete goods and the substitutes condition, if the initial price vector is chosen to be an integer-valued Walrasian equilibrium price vector of the market without bidder $i$, and if each bidder $j \neq i$ bids sincerely, then bidder $i$ maximizes her payoff by bidding sincerely and thereby receives exactly her VCG payoff; and:

THEOREM 4': With discrete goods, the substitutes condition, and any integer-valued initial price vector, sincere bidding by every bidder is an ex post perfect equilibrium of the parallel auction game, prices converge to a Walrasian equilibrium price vector in finitely many iterations, and the outcome is exactly that of the VCG mechanism.

Moreover, in each of Theorems $2^{\prime}$ to $4^{\prime}$, if the initial price vector equals zero (or is below the 
lowest Walrasian equilibrium price vector $\mathbf{p}$ ), then prices converge monotonically (in a nondecreasing direction) to the lowest Walrasian equilibrium price vector $\mathbf{p}$. Similarly, if the initial price vector is above the highest Walrasian equilibrium price vector $\overline{\mathbf{p}}$, then prices converge monotonically (in a nonincreasing direction) to the highest Walrasian equilibrium price vector $\overline{\mathbf{p}}$. These two facts can be shown using Propositions 4 and 5 in Appendix B.

\section{Conclusion}

This article has considered economic environments with $K$ types of heterogeneous commodities, containing consumers with quasilinear utilities and pure private values. It has introduced a dynamic clock auction procedure in which sincere bidding is an equilibrium that yields Walrasian equilibrium price vectors and efficient outcomes. This is shown both for a divisible commodities environment (with strictly concave utility functions) and a discrete commodities environment (with substitutes preferences). Starting from an initial price vector of zero and using a single ascending price trajectory, a "modified" VCG mechanism is implemented; and using $n$ ascending price trajectories that may be elicited in parallel, the full VCG mechanism is implemented. Using an adjustment process that allows prices to increase or decrease, global convergence to a Walrasian equilibrium price vector is obtained.

One immediate question is whether the auction design herein can be generalized to treat the case where bidders have interdependent values. Perry and Reny (2005) provide an affirmative answer to this question for my earlier efficient auction design treating homogeneous goods. They consider a model where each bidder receives a one-dimensional signal and where each bidder's valuation depends on the signals received by all $n$ bidders. They show that: (a) with two bidders, the homogeneous goods auction leads to efficient outcomes with interdependent values; and (b) by allowing bidders to submit directed demands (one against each other bidder), it is possible to obtain efficient outcomes with interdependent values and $n$ bidders.
I conjecture that essentially the same two steps can be replicated for the auction design herein. That is, with interdependent values and two bidders, the efficient dynamic auction for heterogeneous commodities should also lead to efficient outcomes; and, again, by allowing bidders to submit directed demands (one against each other bidder), it should be possible to obtain efficient outcomes with interdependent values and $n$ bidders.

At the same time, such a complication of the current auction design is not entirely in the spirit of the current article. Even the $n$ parallel auctions version of the current design has been critiqued as requiring excessive communication and for requesting bids that may be of minimal payoff relevance to the bidders making them. Introducing directed demands into the design would be a further step in the direction of increasing the required communication and complicating the auction.

Despite their theoretical limitations, simple clean auction designs have distinct advantages over the more complicated mechanisms that are required for achieving full efficiency. These advantages are difficult to model formally and tend not to be treated in the existing literature. Nevertheless, the relatively simple designs of the predecessor and current articles seem likely to fare well on matters of cognitive simplicity and robustness. If bidders are easily able to understand the auction design, they seem more likely to bid consistently with equilibrium behavior. And if an auction design cleanly reflects intuitive first principles, it is more likely to perform robustly in environments somewhat different from stylized economic models.

The viewpoint of the predecessor article for homogeneous goods has been that a good compromise between these competing considerations is to utilize an auction design that is dynamic (so as to give some recognition of value interdependencies) while still simple at every step. The heterogeneous commodities design of the current article attempts to adhere to this philosophy as much as possible. By so doing, it aspires to introduce efficient auction procedures sufficiently transparent and robust that they might someday find themselves adopted into practical usage. 


\section{Appendix A: Proofs of Lemmas and Theorems}

\section{PROOF OF LEMMA 1:}

By Theorem 9-26 of Apostol (1957), since $p^{k}$ is continuous on $[0, T]$ and each $x_{j}^{k}$ (and, hence, $x_{-i}^{k}$ ) is of bounded variation on $[0, T]$, each Stieltjes integral $\int_{0}^{T} p^{k}(t) d x_{-i}^{k}(t)$ exists.

\section{PROOF OF LEMMA 2:}

The Stieltjes integral $\int_{0}^{T} p^{k}(t) d x_{j}^{k}(\mathbf{p}(t))$ exists if and only if the Stieltjes integral $\int_{0}^{T} x_{j}^{k}(\mathbf{p}(t)) d p^{k}(t)$ exists. Consequently, by integration by parts,

$$
\int_{0}^{T} \mathbf{p}(t) \cdot d \mathbf{x}_{\mathbf{j}}(\mathbf{p}(t))=\mathbf{p}(T) \cdot \mathbf{x}_{\mathbf{j}}(\mathbf{p}(T))-\mathbf{p}(0) \cdot \mathbf{x}_{\mathbf{j}}(\mathbf{p}(0))-\int_{0}^{T} \mathbf{x}_{\mathbf{j}}(\mathbf{p}(t)) \cdot d \mathbf{p}(t), \quad \text { for all } j \in N
$$

Let $\Gamma$ denote the (piecewise smooth) curve in $\mathbb{R}^{K}$ described by $\mathbf{p}(t), t \in[0, T]$. The integral on the right side of equation (13) may be rewritten as the line integral $\int_{\Gamma} \mathbf{x}_{\mathbf{j}} \cdot d \mathbf{p}$. (For a formal definition of the line integral, see Apostol, 1957, Definition 10-32.) Since $V_{j}(\cdot)$ is a convex function and $-\mathbf{x}_{\mathbf{j}}(\cdot)$ is a measurable selection from its subdifferential (see equation (3), above, and the surrounding text), Theorem 1 of Vijay Krishna and Eliot Maenner (2001) guarantees that the line integral is independent of path and equals $\int_{\Gamma} \mathbf{x}_{\mathbf{j}} \cdot d \mathbf{p}=-V_{j}(\mathbf{p}(T))+V_{j}(\mathbf{p}(0))$. Noting that $V_{j}(\mathbf{p}(t))=U_{j}\left(\mathbf{x}_{\mathbf{j}}(\mathbf{p}(t))\right)-\mathbf{p}(t) \cdot$ $\mathbf{x}_{\mathbf{j}}(\mathbf{p}(t))$,

$$
\int_{0}^{T} \mathbf{p}(t) \cdot d \mathbf{x}_{\mathbf{j}}(\mathbf{p}(t))=U_{j}\left(\mathbf{x}_{\mathbf{j}}(\mathbf{p}(T))\right)-U_{j}\left(\mathbf{x}_{\mathbf{j}}(\mathbf{p}(0))\right)
$$

and summing over all $j \neq i$ yields equation (8).

\section{PROOF OF LEMMA 3:}

With strictly concave utility functions and compact, convex consumption sets, observe that the demand correspondences are single-valued and continuous in price. Given that equation (9) describing Walrasian tâtonnement is uniformly bounded, all price paths $\mathbf{p}(\cdot)$ are Lipschitz-continuous in $t$, and sincere bidding induces continuous actions $\mathbf{x}_{\mathbf{i}}(\cdot)$ by each bidder that are of bounded variation in $t$ for each coordinate $k$. Hence, Lemmas 1 and 2 apply. Defining the Lyapunov function, $L(\cdot)$, by equation (10) (see, for example, Varian, 1981, pp. 104-06) and applying equation (3), we find that

$$
\dot{L}=\frac{d L(\mathbf{p}(t))}{d t}=\left(\mathbf{S}-\sum_{i=1}^{n} \mathbf{q}_{\mathbf{i}}(\mathbf{p}(t))\right) \cdot \dot{\mathbf{p}}(t), \text { almost everywhere in } t .
$$

Observe that, in the adjustment process of equation (9), $\dot{p}^{k}$ has the opposite sign as $S^{k}-\sum_{i=1}^{n} q_{i}^{k}(\mathbf{p})$. Hence, equation (15) implies that $\dot{L}=0$ at all Walrasian equilibrium price vectors and $\dot{L}<0$ at all other price vectors. Note that $L(\cdot)$ as defined by equation $(10)$ is convex, and so any local minimum is also a global minimum. Letting $L^{*}=\lim _{t \rightarrow \infty} L(\mathbf{p}(t))$, we conclude that $L^{*}$ is the minimum of $L(\cdot)$, and $\mathbf{p}^{*}$ associated with $L^{*}$ is a Walrasian equilibrium price vector.

\section{PROOF OF THEOREM 1:}

By Lemma 2, bidder $i$ 's payoff from any bidding strategy $\sigma_{i} \in \Sigma_{i}$ that causes the auction to terminate at time $T$ with price vector $\mathbf{p}(T)$ and with bidder $i$ receiving quantity $\mathbf{x}_{\mathbf{i}}(T)$ is 


$$
\begin{aligned}
U_{i}\left(\mathbf{x}_{\mathbf{i}}(T)\right)-a_{i}(T) & =U_{i}\left(\mathbf{x}_{\mathbf{i}}(T)\right)-\mathbf{p}(0) \cdot\left[\mathbf{S}-\mathbf{q}_{-\mathbf{i}}(\mathbf{p}(0))\right]+\int_{0}^{T} \mathbf{p}(t) \cdot d \mathbf{q}_{-\mathbf{i}}(\mathbf{p}(t)) \\
& =U_{i}\left(\mathbf{x}_{\mathbf{i}}(T)\right)-\mathbf{p}(0) \cdot\left[\mathbf{S}-\mathbf{q}_{-\mathbf{i}}(\mathbf{p}(0))\right]+U_{-i}\left(\overline{\mathbf{q}}_{-\mathbf{i}}(\mathbf{p}(T))\right)-U_{-i}\left(\overline{\mathbf{q}}_{-\mathbf{i}}(\mathbf{p}(0))\right)
\end{aligned}
$$

In order for the auction to terminate at time $T$, given the opposing bidders' strategies of $\overline{\mathbf{q}}_{-\mathbf{i}}(\mathbf{p}(\cdot))$, bidder $i$ must bid $\mathbf{x}_{\mathbf{i}}(T)=\mathbf{S}-\mathbf{q}_{-\mathbf{i}}(\mathbf{p}(T))$. Consequently, bidder $i$ receives payoff of

$$
U_{i}\left(\mathbf{S}-\mathbf{q}_{-\mathbf{i}}(\mathbf{p}(T))\right)+U_{-i}\left(\overline{\mathbf{q}}_{-\mathbf{i}}(\mathbf{p}(T))\right)-\left\{\mathbf{p}(0) \cdot\left[\mathbf{S}-\mathbf{q}_{-\mathbf{i}}(\mathbf{p}(0))\right]+U_{-i}\left(\overline{\mathbf{q}}_{-\mathbf{i}}(\mathbf{p}(0))\right)\right\} .
$$

Since the expression within braces in expression (17) — determined only by the starting price and the other bidders' starting actions - is a constant, bidder $i$ maximizes expression (17) by maximizing the first two terms. These first two terms coincide with social surplus for the allocation associated with $\mathbf{p}(T)$.

Moreover, given the quasilinearity of utility, the Theorems of Welfare Economics imply that any Walrasian equilibrium is associated with a surplus-maximizing allocation, and vice versa. Consequently, bidder $i$ 's payoff is maximized if and only if a Walrasian equilibrium price vector is the endpoint. By Lemma 3, bidder $i$ can attain this maximum by bidding sincerely.

\section{PROOF OF THEOREM 2:}

Suppose that all opposing bidders $j \neq i$ bid sincerely in the auction game. One available strategy for bidder $i$ is also to bid sincerely. By Lemma 3, price then converges to a Walrasian equilibrium price vector and, by Theorem 1 , the strategy is a best response for bidder $i$. Furthermore, the payoff in expression (17) then evaluates to bidder $i$ 's payoff from the modified VCG mechanism with price of $\mathbf{p}(0)$. This holds for every bidder $i=1, \ldots, n$, proving the theorem.

\section{PROOF OF THEOREM 3:}

Following the proof of Theorem 1, observe that if the initial price is chosen such that the market without bidder $i$ clears at $\mathbf{p}(0)$, then the term $\mathbf{p}(0) \cdot\left[\mathbf{S}-\mathbf{q}_{-\mathbf{i}}(\mathbf{p}(0))\right]$ in expression (17) equals zero and, by the First Theorem of Welfare Economics, the term $U_{-i}\left(\overline{\mathbf{q}}_{-\mathbf{i}}(\mathbf{p}(0))\right)$ in expression (17) equals $W_{-i}^{*}$ of Definition 2. The remaining payoff term, when maximized, is thus exactly bidder $i$ 's payoff from the VCG mechanism.

\section{PROOF OF THEOREM 4:}

First, let us consider the full parallel auction game in which all $2 n$ steps are carried out. Observe that the determination of $\mathbf{p}_{-\mathbf{i}}$ has no relevance to the payoff of any bidder except for bidder $i$, and bidder $i$ 's demand reports are ignored in the price adjustment of step $i$. Hence, by Lemma 3, sincere bidding at each step $i=1, \ldots, n$ is a best response for every bidder, yielding a Walrasian equilibrium price vector $\mathbf{p}_{-\mathbf{i}}$ for the economy without bidder $i$. Meanwhile, the same reasoning as in the proof of Theorem 2 applies at each step $i=n+1, \ldots, 2 n$, so starting from price vector $\mathbf{p}_{-\mathbf{i}}$, sincere bidding is a best response for every bidder, yielding a Walrasian equilibrium price vector $\mathbf{p}^{*}$ for the economy with all bidders. Further observe that the results of steps $n+1, \ldots, 2 n$ are consistent with one another (i.e., they yield the same allocations), and the payment of each bidder $i$ is given by the line integral of equation (7) along the path from $\mathbf{p}_{-\mathbf{i}}$ to $\mathbf{p}^{*}$. As in Theorem 3, it equals exactly the VCG payment of bidder $i$.

Second, let us consider the shortcut procedure which, without loss of generality, consists of steps $1, \ldots, n$ and step $2 n$. As in the previous paragraph, at each step $i=1, \ldots, n-1$, the determination of $\mathbf{p}_{-\mathbf{i}}$ has no relevance to the payoff of any bidder except for bidder $i$, and so sincere bidding is an equilibrium yielding a price vector $\mathbf{p}_{-\mathbf{i}}$. At step $n$, bidder $i(1 \leq i \leq n-1)$ now has the capability and potential incentive for manipulating the determination of price vector $\mathbf{p}_{-\mathbf{n}}$, since her payoff is 
now calculated through $\mathbf{p}_{-\mathbf{n}}$. Observe, however, that bidder $i$ 's payment is given by the line integral of equation (7) along the union of three paths: the path from $\mathbf{p}_{-\mathbf{i}}$ to $\mathbf{p}(0)$; the path from $\mathbf{p}(0)$ to $\mathbf{p}_{-\mathbf{n}}$; and the path from $\mathbf{p}_{-\mathbf{n}}$ to $\mathbf{p}^{*}$. Consequently, by Lemma 2, if each opposing bidder bids sincerely according to a strictly concave utility function, the line integral along the union of the three paths is path independent and evaluates the same as in step $n+i$ of the previous paragraph. In particular, it does not depend on $\mathbf{p}_{-\mathbf{n}}$ and, by the same reasoning as in the proof of Theorem 1 , bidder $i$ maximizes her payoff by selecting a Walrasian equilibrium price vector $\mathbf{p}^{*}$ as the endpoint. Thus, sincere bidding at steps $n$ and $2 n$ is a best response and yields bidder $i$ her VCG payoff. Finally, bidder $n$ 's payoff is determined in exactly the same way as in the full parallel auction game and so, as in the previous paragraph, sincere bidding at steps $n$ and $2 n$ is also a best response for bidder $n$.

\section{PROOF OF THEOREM 2':}

By the Global Convergence Theorem of the companion paper (Ausubel, 2005) restated in Appendix B, the global Walrasian tâtonnement algorithm converges from any arbitrary integervalued initial price vector $\mathbf{p}(0)$ to a Walrasian equilibrium price vector in finitely many iterations.

The proof concludes similarly to the proof of Theorem 2 .

\section{Appendix B: Tâtonnement Algorithm for Discrete Goods}

This Appendix outlines the tâtonnement algorithm for discrete goods that is utilized in Section VII of the article. A full development of the algorithm and its properties, including complete proofs, may be found in a companion paper (Ausubel, 2005).

The first step in the argument developing a tâtonnement algorithm establishes that, under Assumptions $\left(\mathrm{A} 1^{\prime}\right)-\left(\mathrm{A} 3^{\prime}\right)$, if a Walrasian equilibrium exists, then Walrasian equilibrium price vectors are associated with minima of the Lyapunov function in equation (10). A related duality result concerning equation (10) was first identified by Milgrom (2004, equation (8.8)). In the companion paper, a complete proof is provided of Milgrom's insight, and the result is then utilized to prove:

PROPOSITION 1: In the discrete goods model with Assumptions (A1')-(A3'), suppose that a Walrasian equilibrium exists. Then the set of Walrasian equilibrium price vectors equals the arg min of $L(\cdot)$ defined by equation (10), and the set of Walrasian equilibria equals the set of all $\left(\mathbf{p}^{*}, \mathbf{x}^{*}\right)$ such that $\mathbf{p}^{*}$ minimizes $L(\cdot)$ and $\left(\mathbf{x}_{\mathbf{1}}^{*}, \ldots, \mathbf{x}_{\mathbf{n}}^{*}\right)$ maximizes $\sum_{i=1}^{n} U_{i}\left(\mathbf{x}_{\mathbf{i}}\right)$ subject to $\mathbf{x}_{\mathbf{i}} \in X_{i}$, for all $i=1, \ldots$, $n$, and $\sum_{i=1}^{n} \mathbf{x}_{\mathbf{i}}=\mathbf{S}$.

Since the addition of the substitutes condition (A4') guarantees the existence of Walrasian equilibrium (Kelso and Crawford, 1982), making Assumptions (A1')-(A4') assures that the hypothesis of Proposition 1 is satisfied. In turn, the substitutes condition (A4') is provided a convenient characterization by Ausubel and Milgrom (2002, Theorem 10): commodities are substitutes for bidder $i$ if and only if the indirect utility function, $V_{i}(\cdot)$, is a submodular function. At the same time, even without the substitutes condition, the indirect utility function is convex. Given that the Lyapunov function $L(\cdot)$ is the sum of a linear function and the indirect utility functions of the $n$ bidders, it follows that $L(\cdot)$ is a submodular function, as well as a convex function.

Recall that $L(\cdot)$ is defined to be a submodular function if, for all elements $\mathbf{p}$ and $\mathbf{p}^{\prime}$ of the domain, the following inequality holds:

$$
L\left(\mathbf{p} \vee \mathbf{p}^{\prime}\right)+L\left(\mathbf{p} \wedge \mathbf{p}^{\prime}\right) \leq L(\mathbf{p})+L\left(\mathbf{p}^{\prime}\right),
$$

where $\mathbf{p} \vee \mathbf{p}^{\prime}$ denotes the coordinate-by-coordinate maximum of $\mathbf{p}$ and $\mathbf{p}^{\prime}$, and $\mathbf{p} \wedge \mathbf{p}^{\prime}$ denotes the coordinate-by-coordinate minimum. Let $\mathbf{p}$ and $\mathbf{p}^{\prime}$ be any two minimizers of $L(\cdot)$. From inequality (18), observe that $L\left(\mathbf{p} \vee \mathbf{p}^{\prime}\right) \leq L(\mathbf{p})$ and $L\left(\mathbf{p} \wedge \mathbf{p}^{\prime}\right) \leq L(\mathbf{p})$; that is, $\mathbf{p} \vee \mathbf{p}^{\prime}$ and $\mathbf{p} \wedge \mathbf{p}^{\prime}$ are also 
minimizers of $L(\cdot)$. Thus, the set of minimizers of $L(\cdot)$ over the lattice of price vectors is itself a sublattice. One can further show that, if the data of the problem are integers, then the lowest and highest minimizers are also integers. (See the companion paper or apply Proposition 3.) We have:

COROLLARY TO PROPOSITION 1: In the discrete goods model with Assumptions (A1')-(A4'), the set of Walrasian equilibrium price vectors is a nonempty lattice and the lowest and highest Walrasian equilibrium price vectors, $\mathbf{p}$ and $\overline{\mathbf{p}}$, consist of integers.

Suppose that bidder $i$ reports her demand correspondence $Q_{i}(\mathbf{p})$ evaluated at an integer-valued price vector $\mathbf{p}$. There is a simple procedure that can be used to identify candidate optimal commodity bundles in a neighborhood of $\mathbf{p}$.

DEFINITION 5: Let $\mathbf{p}$ be any integer-valued price vector, and let $Q_{i}(\mathbf{p})$ be bidder i's demand correspondence evaluated at $\mathbf{p}$. Let $\mathbf{\Delta}$ be any price vector belonging to the unit $K$-dimensional cube $\{\boldsymbol{\Delta}: \mathbf{0} \leq \boldsymbol{\Delta} \leq \mathbf{1}\}$, where the symbol $\mathbf{0}$ denotes the $K$-dimensional vector $(0, \ldots, 0)$ and the symbol 1 denotes the $K$-dimensional vector $(1, \ldots, 1)$. Select:

$$
\tilde{\mathbf{x}}_{\mathbf{i}} \in \arg \min _{\mathbf{x} \in Q_{i}(\mathbf{p})}\{\boldsymbol{\Delta} \cdot \mathbf{x}\} \quad \text { and } \quad \tilde{\mathbf{y}}_{\mathbf{i}} \in \arg \max _{\mathbf{x} \in Q_{i}(\mathbf{p})}\{\boldsymbol{\Delta} \cdot \mathbf{x}\}
$$

In other words, $\tilde{\mathbf{x}}_{\mathbf{i}}$ is selected to be an element of $Q_{i}(\mathbf{p})$ that increases in cost the least as the price vector rises from $\mathbf{p}$ to $\mathbf{p}+\boldsymbol{\Delta}$, and $\tilde{\mathbf{y}}_{\mathbf{i}}$ is selected to be an element of $Q_{i}(\mathbf{p})$ that decreases in cost the most as the price vector falls from $\mathbf{p}$ to $\mathbf{p}-\mathbf{\Delta}$.

Given the integer values assumed in Assumption (A1'), observe that a small change in prices (i.e., a price change causing a bidder's total payment to increase or decrease by less than one) from an integer-valued price vector cannot add any new commodity bundle into the demand correspondence, implying that $\tilde{\mathbf{x}}_{\mathbf{i}}$ and $\tilde{\mathbf{y}}_{\mathbf{i}}$ of equation (19) are optimal commodity bundles. Gul and Stacchetti (1999, Lemma 2) show that substitutes preferences imply the single-improvement property: if $\mathbf{x}_{\mathbf{i}}$ is not optimal for bidder $i$ at a given price, then there exists an alternative commodity bundle $\mathbf{x}_{\mathbf{i}}^{\prime}$ such that $\#\left(\mathbf{x}_{\mathbf{i}} \mid \mathbf{x}_{\mathbf{i}}^{\prime}\right) \leq 1, \#\left(\mathbf{x}_{\mathbf{i}}^{\prime} \mid \mathbf{x}_{\mathbf{i}}\right) \leq 1$, and bidder $i$ strictly prefers $\mathbf{x}_{\mathbf{i}}^{\prime}$ to $\mathbf{x}_{\mathbf{i}}$. (That is, $\mathbf{x}_{\mathbf{i}}^{\prime}$ is formed by adding at most one good and subtracting at most one good from $\mathbf{x}_{\mathbf{i}}$.) The single-improvement property may be exploited to establish a very powerful result: for a bidder with substitutes preferences, $\tilde{\mathbf{x}}_{\mathbf{i}}$ continues to be optimal provided that each price is increased by no more than one and $\tilde{\mathbf{y}}_{\mathbf{i}}$ continues to be optimal provided that each price is decreased by no more than one. We have the following result, which is central to both the tâtonnement algorithm and its application to an efficient dynamic auction, and so its proof is also included:

PROPOSITION 2: If Assumptions (A1')-(A4') hold for bidder i, then $\tilde{\mathbf{x}}_{\mathbf{i}}$ and $\tilde{\mathbf{y}}_{\mathbf{i}}$ of equation (19) satisfy:

$$
\tilde{\mathbf{x}}_{\mathbf{i}} \in \mathrm{Q}_{\mathrm{i}}(\mathbf{p}+\lambda \boldsymbol{\Delta}) \quad \text { and } \quad \tilde{\mathbf{y}}_{\mathrm{i}} \in \mathrm{Q}_{\mathrm{i}}(\mathbf{p}-\lambda \boldsymbol{\Delta}) \text {, for all scalars } \lambda>0 \text { such that } \mathbf{0} \leq \lambda \boldsymbol{\Delta} \leq \mathbf{1} .
$$

\section{PROOF OF PROPOSITION 2:}

Suppose to the contrary that there exists $\lambda>0$ such that $\mathbf{0} \leq \lambda \boldsymbol{\Delta} \leq \mathbf{1}$ but $\tilde{\mathbf{x}}_{\mathbf{i}} \notin Q_{i}(\mathbf{p}+\lambda \boldsymbol{\Delta})$. By the single-improvement property, there exists $\mathbf{x}_{\mathbf{i}}^{\prime}$ such that $\#\left(\tilde{\mathbf{x}}_{\mathbf{i}} \mid \mathbf{x}_{\mathbf{i}}^{\prime}\right) \leq 1$, \#( $\left.\left(\mathbf{x}_{\mathbf{i}}^{\prime}\right) \tilde{\mathbf{x}}_{\mathbf{i}}\right) \leq 1$, and bidder $i$ strictly prefers $\mathbf{x}_{\mathbf{i}}^{\prime}$ to $\tilde{\mathbf{x}}_{\mathbf{i}}$ at $\mathbf{p}+\lambda \boldsymbol{\Delta}$. By construction, $\tilde{\mathbf{x}}_{\mathbf{i}}$ is optimal at $\mathbf{p}+\lambda \boldsymbol{\Delta}$ among all elements of $Q_{i}(\mathbf{p})$; consequently, $\mathbf{x}_{\mathbf{i}}^{\prime} \notin Q_{i}(\mathbf{p})$. Given the assumption included in (A1') that bidders' values are integers, this means that $U_{i}\left(\mathbf{x}_{\mathbf{i}}^{\prime}\right)-\mathbf{p} \cdot \mathbf{x}_{\mathbf{i}}^{\prime} \leq U_{i}\left(\tilde{\mathbf{x}}_{\mathbf{i}}\right)-\mathbf{p} \cdot \tilde{\mathbf{x}}_{\mathbf{i}}-1$. Meanwhile, since $\mathbf{0} \leq \lambda \boldsymbol{\Delta} \leq \mathbf{1}$ and $\#\left(\tilde{\mathbf{x}}_{\mathbf{i}} \mid \mathbf{x}_{\mathbf{i}}^{\prime}\right) \leq 1$, we necessarily have $\lambda \boldsymbol{\Delta} \cdot \tilde{\mathbf{x}}_{\mathbf{i}} \leq \lambda \boldsymbol{\Delta} \cdot \mathbf{x}_{\mathbf{i}}^{\prime}+1$. Combining these two inequalities, we conclude that $U_{i}\left(\mathbf{x}_{\mathbf{i}}^{\prime}\right)-(\mathbf{p}+\lambda \boldsymbol{\Delta}) \cdot \mathbf{x}_{\mathbf{i}}^{\prime} \leq U_{i}\left(\tilde{\mathbf{x}}_{\mathbf{i}}\right)-(\mathbf{p}+\lambda \boldsymbol{\Delta}) \cdot \tilde{\mathbf{x}}_{\mathbf{i}}$, contradicting that bidder $i$ prefers 
$\mathbf{x}_{\mathbf{i}}^{\prime}$ to $\tilde{\mathbf{x}}_{\mathbf{i}}$ at $\mathbf{p}+\lambda \boldsymbol{\Delta}$. An analogous contradiction is obtained if there exists $\lambda>0$ such that $\mathbf{0} \leq \lambda \boldsymbol{\Delta}$ $\leq \mathbf{1}$ but $\tilde{\mathbf{y}}_{\mathbf{i}} \notin Q_{i}(\mathbf{p}-\lambda \boldsymbol{\Delta})$.

The third step in the argument developing a tâtonnement algorithm is to define our price adjustment rules. Given a current price vector $\mathbf{p}(t)$, the auctioneer asks each bidder $i$ to report its demand correspondence $Q_{i}(\cdot)$ evaluated at $\mathbf{p}(t)$. Using equation (19) and Proposition 2, the auctioneer can extend the report to identify an optimal commodity bundle at every point in the unit $K$ dimensional cubes $\{\mathbf{p}+\boldsymbol{\Delta}: \mathbf{0} \leq \boldsymbol{\Delta} \leq \mathbf{1}\}$ and $\{\mathbf{p}-\boldsymbol{\Delta}: \mathbf{0} \leq \boldsymbol{\Delta} \leq \mathbf{1}\}$. While the demand that may be selected at a particular price vector is not necessarily unique, all optimal demands yield the same indirect utility, so that the indirect utility function $V_{i}(\cdot)$ of each bidder $i$, once specified at $\mathbf{p}(t)$, has a unique extension to the unit $K$-dimensional cubes. Consequently, the Lyapunov function $L(\cdot)$ of equation (10) also has a unique extension.

The auctioneer then determines the price vector on the lattice $\{\mathbf{p}+\boldsymbol{\Delta}: \mathbf{0} \leq \boldsymbol{\Delta} \leq \mathbf{1}\}$ that minimizes the Lyapunov function $L(\cdot)$ and uses this as the next price vector, $\mathbf{p}(t+1)$. This will be the ascending price adjustment rule. Alternatively, the auctioneer determines the price vector on the lattice $\{\mathbf{p}-$ $\boldsymbol{\Delta}: \mathbf{0} \leq \boldsymbol{\Delta} \leq \mathbf{1}\}$ that minimizes $L(\cdot)$. This will be the descending price adjustment rule.

In general, the Lyapunov function $L(\cdot)$ is likely to have multiple minimizers. In this event, the auctioneer selects among them a minimal minimizer, for the ascending rule, or a maximal minimizer, for the descending rule. We define the minimal and maximal minimizers as follows:

DEFINITION 6 (minimal and maximal minimizers): Given integer-valued price vector $\mathbf{p}$ and Lyapunov function $L(\cdot)$ defined on $\{\mathbf{p}+\boldsymbol{\Delta}: \mathbf{0} \leq \boldsymbol{\Delta} \leq \mathbf{1}\}$, a minimal minimizer $\mathbf{p}_{+}$is defined by

$$
\mathbf{p}_{+} \in \arg \min _{\hat{\mathbf{p}} \in\{\mathbf{p}+\boldsymbol{\Delta}: \mathbf{0} \leq \boldsymbol{\Delta} \leq \mathbf{1}\}}\{L(\hat{\mathbf{p}})\},
$$

with the property that, for any $\mathbf{p}^{\prime}$ such that $\mathbf{p} \leq \mathbf{p}^{\prime} \leq \mathbf{p}_{+}$and $\mathbf{p}^{\prime} \neq \mathbf{p}_{+}$, we have $L\left(\mathbf{p}^{\prime}\right)>L\left(\mathbf{p}_{+}\right)$. Given Lyapunov function $L(\cdot)$ defined on $\{\mathbf{p}-\boldsymbol{\Delta}: \mathbf{0} \leq \boldsymbol{\Delta} \leq \mathbf{1}\}$, a maximal minimizer $\mathbf{p}_{-}$is defined by

$$
\mathbf{p}_{-} \in \arg \min _{\hat{\mathbf{p}} \in\{\mathbf{p}-\mathbf{\Delta}: \mathbf{0} \leq \boldsymbol{\Delta} \leq \mathbf{1}\}}\{L(\hat{\mathbf{p}})\}
$$

with the property that, for any $\mathbf{p}^{\prime}$ such that $\mathbf{p}_{-} \leq \mathbf{p}^{\prime} \leq \mathbf{p}$ and $\mathbf{p}^{\prime} \neq \mathbf{p}_{-}$, we have $L\left(\mathbf{p}^{\prime}\right)>L\left(\mathbf{p}_{-}\right)$.

Thus, in Definition 6, the price vector $\mathbf{p}_{+}$is a minimal minimizer in the sense that it minimizes the Lyapunov function $L(\cdot)$ and there is no alternative price vector, at least as small in every coordinate, that yields as low a value. The price vector $\mathbf{p}_{-}$is a maximal minimizer in the sense that it minimizes the Lyapunov function $L(\cdot)$ and there is no alternative price vector, at least as large in every coordinate, that yields as low a value. Since the Lyapunov function $L(\cdot)$ is submodular under $\left(\mathrm{A} 4^{\prime}\right)$ and truthful reporting, the set of minimizers is a sublattice and there is a (unique) lowest and highest minimizer. Moreover, application of Proposition 2 allows us to demonstrate that the minimal and maximal minimizers are integer valued. In the companion paper, we prove the following result:

PROPOSITION 3: In the discrete goods model with Assumptions (A1')-(A4') and truthful reporting by bidders, the minimal minimizer $\mathbf{p}_{+}$of equation (21) and the maximal minimizer $\mathbf{p}_{-}$of equation (22) are uniquely defined and integer valued.

In light of Proposition 3, it is unnecessary to search everywhere in the unit $K$-dimensional cubes $\{\mathbf{p}+\boldsymbol{\Delta}: \mathbf{0} \leq \boldsymbol{\Delta} \leq \mathbf{1}\}$ and $\{\mathbf{p}-\boldsymbol{\Delta}: \mathbf{0} \leq \boldsymbol{\Delta} \leq \mathbf{1}\}$ for minima. It is sufficient to search only among vertices of the unit $K$-dimensional cubes and one would be guaranteed of achieving the same minimum in the Lyapunov function. This motivates the actual definition that we will use in specifying the tâtonnement process: 
DEFINITION 7 (minimal and maximal minimizers among vertices): For $E \subset\{1, \ldots, K\}$, let $\mathbf{1}^{E}$ denote the price vector whose $k^{\text {th }}$ coordinate equals 1, for $k \in E$, and equals 0, for $k \notin E$. Given integer-valued price vector $\mathbf{p}(t)$ and Lyapunov function $L(\cdot)$ defined on $\{\mathbf{p}(t)+\boldsymbol{\Delta}: \mathbf{0} \leq \boldsymbol{\Delta} \leq \mathbf{1}\}$ and $\{\mathbf{p}(t)-\boldsymbol{\Delta}: \mathbf{0} \leq \boldsymbol{\Delta} \leq \mathbf{1}\}$, define the functions

$$
\ell_{+}(E)=L\left(\mathbf{p}(t)+\mathbf{1}^{E}\right) \quad \text { and } \quad \ell_{-}(E)=L\left(\mathbf{p}(t)-\mathbf{1}^{E}\right)
$$

In the ascending price adjustment rule, the next price vector is a minimal minimizer defined by $\mathbf{p}(t+1)=\mathbf{p}(t)+\mathbf{1}^{E_{+}}$, where $E_{+}$is defined by

$$
E_{+} \in \arg \min _{\hat{E} \subset\{1, \ldots, K\}}\left\{\ell_{+}(\hat{E})\right\},
$$

with the property that, for any $E^{\prime}$ which is a strict subset of $E_{+}$, we have $\ell_{+}\left(E^{\prime}\right)>\ell_{+}\left(E_{+}\right)$. In the descending price adjustment rule, the next price vector is a maximal minimizer defined by $\mathbf{p}(t+1)=$ $\mathbf{p}(t)-\mathbf{1}^{E_{-}}$, where $E_{-}$is defined by

$$
E_{-} \in \arg \min _{\hat{E} \subset\{1, \ldots, K\}}\left\{\ell_{-}(\hat{E})\right\},
$$

with the property that, for any $E^{\prime}$ which is a strict subset of $E_{-}$, we have $\ell_{-}\left(E^{\prime}\right)>\ell_{-}\left(E_{-}\right)$.

Thus, in Definition 7, the set $E_{+}$is a minimal minimizing set in the sense that $E_{+}$minimizes the Lyapunov function $\ell_{+}(\cdot)$ and there is no strict subset that yields as low a value. The set $E_{-}$is also a minimal minimizing set in the sense that $E_{-}$minimizes the Lyapunov function $\ell_{-}(\cdot)$ and there is no strict subset that yields as low a value. However, the implied price, $\mathbf{p}(t+1)=\mathbf{p}(t)-\mathbf{1}^{E_{-}}$, is a maximal minimizer, since there is no alternative price vector, at least as great in every coordinate, that yields as low a value.

Even if reports are not truthful, the minimization problems of equations (24) and (25) are each guaranteed to have at least one minimal minimizer, since the respective domains (each comprising the set of all subsets of commodities) are finite. In this event, if the minimization problem has more than one minimal minimizer, the auctioneer arbitrarily selects any one.

In the tâtonnement algorithm, the price adjustment rule of Definition 7 will be applied iteratively. It continues so long as $\mathbf{p}(t+1) \neq \mathbf{p}(t)$ and it terminates at the first time $T$ such that $\mathbf{p}(T+1)=\mathbf{p}(T)$. The fourth step in the argument developing the tâtonnement algorithm is to show that the ascending price adjustment rule terminates at $\mathbf{p}(T) \geq \mathbf{p}$. Otherwise, the submodularity of $L(\cdot)$ allows one to construct $\mathbf{p}^{\prime} \geq \mathbf{p}(T)$ such that $L\left(\mathbf{p}^{\prime}\right)<L(\mathbf{p}(\bar{T}))$, and the convexity of $L(\cdot)$ guarantees that such a $\mathbf{p}^{\prime}$ exists in a neighborhood of $\mathbf{p}(T)$, contradicting that the adjustment rule terminated at time $T$. Similarly, the descending price adjustment rule terminates at $\mathbf{p}(T) \leq \overline{\mathbf{p}}$. We have:

PROPOSITION 4: For the ascending price adjustment rule, if $\mathbf{p}(T+1)=\mathbf{p}(T)$ then $\mathbf{p}(T) \geq \underline{\mathbf{p}}$. For the descending price adjustment rule, if $\mathbf{p}(T+1)=\mathbf{p}(T)$ then $\mathbf{p}(T) \leq \overline{\mathbf{p}}$.

The fifth and final step in the argument developing a tâtonnement algorithm is to show that the ascending (descending) price adjustment rule never "overshoots" the lowest (highest) Walrasian equilibrium price vector. The proof, which can be found in the companion paper, follows by supposing that there exists price vector $\mathbf{p}(t)$ such that $\mathbf{p}(t) \leq \mathbf{p}$ but $p(t+1)^{k}>p^{k}$ for some coordinate $k(1 \leq k \leq K)$. Then, it can be shown as a consequence of submodularity that $\mathbf{p}(t+1) \wedge \mathbf{p}$ yields as low a value for $L(\cdot)$ as $\mathbf{p}(t+1)$, contradicting that $\mathbf{p}(t+1)$ is a minimal minimizer. An analogous argument establishes the result for the descending adjustment rule. We have:

PROPOSITION 5: For the ascending price adjustment rule, if $\mathbf{p}(t) \leq \mathbf{p}$, then $\mathbf{p}(t+1) \leq \mathbf{p}$. For the descending price adjustment rule, if $\mathbf{p}(t) \geq \overline{\mathbf{p}}$, then $\mathbf{p}(t+1) \geq \overline{\mathbf{p}}$. 
The global Walrasian tâtonnement algorithm is specified in Section VII. Using Propositions 1-5 and noting that the Lyapunov function is integer-valued and decreases by a positive integer amount at every iteration, the following main result is established in the companion paper:

GLOBAL CONVERGENCE THEOREM: In the discrete goods model with Assumptions (A1')(A4'), starting from any integer-valued initial price vector and given truthful reporting by agents, the global Walrasian tâtonnement algorithm converges to a Walrasian equilibrium price vector in finitely many iterations.

\section{REFERENCES}

Apostol, Tom M. Mathematical analysis: A modern approach to advanced calculus. Reading, MA: Addison-Wesley, 1957.

Arrow, Kenneth J. "Toward a Theory of Price Adjustment," in Moses Abramovitz, ed., The allocation of economic resources. Stanford: Stanford University Press, 1959, pp. 41-51.

Arrow, Kenneth J.; Block, Henry D. and Hurwicz, Leonid. "On the Stability of the Competitive Equilibrium, II." Econometrica, 1959, 27(1), pp. 82-109.

Arrow, Kenneth J. and Hahn, Frank H. General competitive analysis. San Francisco: HoldenDay Publisher, 1971.

Aumann, Robert J. "Markets with a Continuum of Traders." Econometrica, 1964, 32(1-2), pp. 39-50.

Ausubel, Lawrence M. "A Mechanism Generalizing the Vickrey Auction." Unpublished Paper, 1999.

Ausubel, Lawrence M. "System and Method for an Efficient Dynamic Auction for Multiple Objects." U.S. Patent No. 6,026,383, filed January 4, 1996, and issued February 15, 2000.

Ausubel, Lawrence M. "System and Method for an Efficient Dynamic Multi-Unit Auction." U.S. Patent Application No. 09/898,483. Publication No. US 2002/0052828A1, published May 2, 2002.

Ausubel, Lawrence M. "An Efficient AscendingBid Auction for Multiple Objects." University of Maryland, Working Paper No. 97-06, June 1997.

Ausubel, Lawrence M. "An Efficient AscendingBid Auction for Multiple Objects." American Economic Review, 2004, 94(5), pp. 1452-75.

Ausubel, Lawrence M. "Walrasian Tâtonnement for Discrete Goods." Unpublished Paper, 2005.

Ausubel, Lawrence M. and Cramton, Peter. "De- mand Reduction and Inefficiency in MultiUnit Auctions." University of Maryland, Department of Economics, Working Paper No. 96-07, July 2002.

Ausubel, Lawrence M. and Cramton, Peter. "Auctioning Many Divisible Goods." Journal of the European Economic Association, 2004a, 2(2-3), pp. 480-93.

-Ausubel, Lawrence M. and Cramton, Peter. "Vickrey Auctions with Reserve Pricing." Economic Theory, 2004b, 23(3), pp. 493505.

Ausubel, Lawrence M. and Milgrom, Paul R. "Ascending Auctions with Package Bidding." Frontiers of Theoretical Economics, 2002, 1(1), Article 1.

Banks, Jeffrey S.; Ledyard, John O. and Porter, David P. "Allocating Uncertain and Unresponsive Resources: An Experimental Approach." RAND Journal of Economics, 1989, 20(1), pp. 1-25.

Bikhchandani, Sushil; de Vries, Sven; Schummer, James and Vohra, Rakesh V. "Linear Programming and Vickrey Auctions," in Brenda Dietrich and Rakesh V. Vohra, eds., Mathematics of the Internet: E-auction and markets. New York: Springer Verlag, 2002, pp. 75-115.

Bikhchandani, Sushil and Mamer, John W. "Competitive Equilibrium in an Exchange Economy with Indivisibilities." Journal of Economic Theory, 1997, 74(2), pp. 385-413.

Bikhchandani, Sushil and Ostroy, Joseph M. "The Package Assignment Model." Journal of Economic Theory, 2002, 107(2), pp. 377406.

Bikhchandani, Sushil and Ostroy, Joseph M. "Ascending Price Vickrey Auctions." Games and Economic Behavior (forthcoming).

Clarke, Edward H. "Multipart Pricing of Public Goods." Public Choice, 1971, 11, pp. 17-33.

Crémer, Jacques and McLean, Richard P. "Optimal Selling Strategies under Uncertainty for 
a Discriminating Monopolist When Demands Are Interdependent." Econometrica, 1985, 53(2), pp. 345-61.

Dasgupta, Partha and Maskin, Eric S. "Efficient Auctions." Quarterly Journal of Economics, 2000, 115(2), pp. 341-88.

Demange, Gabrielle; Gale, David and Sotomayor, Marilda. "Multi-Item Auctions." Journal of Political Economy, 1986, 94(4), pp. 863-72.

Groves, Theodore. "Incentives in Teams." Econometrica, 1973, 41(4), pp. 617-31.

Gul, Faruk and Stacchetti, Ennio. "Walrasian Equilibrium with Gross Substitutes." Journal of Economic Theory, 1999, 87(1), pp. 95124.

Gul, Faruk and Stacchetti, Ennio. "The English Auction with Differentiated Commodities." Journal of Economic Theory, 2000, 92(1), pp. 66-95.

Hahn, Frank H. "Stability," in Kenneth J. Arrow and Michael D. Intriligator, eds., Handbook of mathematical economics, volume II. Amsterdam: North-Holland, 1982, chap. 16.

Jehiel, Philippe and Moldovanu, Benny. "Efficient Design with Interdependent Valuations." Econometrica, 2001, 69(5), pp. 1237 59.

Kelso, Alexander S., Jr. and Crawford, Vincent P. "Job Matching, Coalition Formation, and Gross Substitutes." Econometrica, 1982, 50(6), pp. 1483-1504.

Krishna, Vijay and Maenner, Eliot. "Convex Potentials with an Application to Mechanism Design." Econometrica, 2001, 69(4), pp. 1113-19.

Maskin, Eric S. "Auctions and Privatization," in Horst Siebert, ed., Privatization: Symposium in honor of Herbert Giersch. Tubingen: Mohr (Siebeck), 1992, pp. 115-36.

Milgrom, Paul. "Putting Auction Theory to
Work: The Simultaneous Ascending Auction." Journal of Political Economy, 2000, 108(2), pp. 245-72.

Milgrom, Paul. Putting auction theory to work. Cambridge: Cambridge University Press, 2004.

Parkes, David C. and Ungar, Lyle H. "Interative Combinational Auctions: Theory and Practice," in American Association of Artificial Intelligence, ed., Proceedings of the 17th $\mathrm{Na}$ tional Conference on Artificial Intelligence (AAAI 2000). Menlo Park: AAI Press, 2000, pp. 74-81.

Perry, Motty and Reny, Philip J. "An Efficient Auction." Econometrica, 2002, 70(3), pp. 1199-1212.

Perry, Motty and Reny, Philip J. "An Efficient Multi-Unit Ascending Auction." Review of Economic Studies, 2005, 72(2), pp. 567-92.

Rockafellar, R. Tyrrell. Convex analysis. Princeton: Princeton University Press, 1970.

Samuelson, Paul A. "The Stability of Equilibrium: Comparative Statics and Dynamics." Econometrica, 1941, 9(2), pp. 97-120.

Scarf, Herbert E. "Some Examples of Global Instability of the Competitive Equilibrium." International Economic Review, 1960, 1(3), pp. 157-72.

Varian, Hal R. "Dynamical Systems with Applications to Economics," in Kenneth J. Arrow and Michael D. Intriligator, eds., Handbook of mathematical economics, volume I. Amsterdam: North-Holland, 1981, chap. 3.

Vickrey, William. "Counterspeculation, Auctions, and Competitive Sealed Tenders." Journal of Finance, 1961, 16(1), pp. 8-37.

Walras, Leon. Éléments d'économie politique pure; ou, théorie de la richesse sociale (Elements of pure economics; or, the theory of social wealth). Lausanne: Corbaz, 1874. 